Piper and Struyvenberg et al.

\title{
A second specificity-determining loop in Class A sortases: Biochemical characterization of natural sequence variation in chimeric SrtA enzymes
}

by

Isabel M. Piper, ${ }^{1 \dagger}$ Sarah A. Struyvenberg, ${ }^{1 \ddagger}$ Jordan D. Valgardson, ${ }^{1 \dagger}$ D. Alex Johnson, ${ }^{1}$ Melody Gao, ${ }^{1}$ Katherine Johnston, ${ }^{1}$ Justin E. Svendsen, ${ }^{1}$ Hanna M. Kodama, ${ }^{1}$ Kelli L. Hvorecny, ${ }^{2}$ John M. Antos, ${ }^{1,}$ Jeanine F. Amacher ${ }^{1, *}$

${ }^{1}$ Department of Chemistry, Western Washington University, Bellingham, WA, 98225

${ }^{2}$ Department of Biochemistry, University of Washington, Seattle, WA, 98195

\$These authors contributed equally to this work.

†Present address: Department of Chemical Systems Biology, Stanford University, Palo Alto, CA, 94305.

*Corresponding Authors:

John Antos, Department of Chemistry, Western Washington University, 516 High St - MS9150, Bellingham, WA, 98225, Tel: +1-360-650-2271, Fax: +1-360-650-2826

Email: antosj@wwu.edu

Jeanine Amacher, Department of Chemistry, Western Washington University, 516 High St MS9150, Bellingham, WA, 98225, Tel: +1-360-650-4397, Fax: +1-360-650-2826

Email: amachej@wwu.edu

Running title: Selectivity determinants in Class A sortases

Keywords: sortases, enzymes, target selectivity, structural biology, protein biochemistry, protein engineering 
Piper and Struyvenberg et al.

\begin{abstract}
Gram-positive bacteria contain sortase enzymes on their cell surfaces that catalyze transpeptidation reactions critical for proper cellular function. In vitro, sortases are used in sortase-mediated ligation (SML) reactions for a variety of protein engineering applications. Historically, sortase A from Staphylococcus aureus (saSrtA) has been the enzyme of choice for SML reactions. However, the stringent specificity of saSrtA for the sequence motif LPXTG limits its uses. Here, we use principal component analysis to identify a structurally conserved loop with a high degree of variability in all classes of sortases. We investigate the contribution of this $\beta 7-\beta 8$ loop, located between the catalytic cysteine and arginine residues and immediately adjacent to the target binding cleft, by designing and testing chimeric sortase enzymes. Our chimeras utilize natural sequence variation of Class A sortases from 8 species engineered into the SrtA sequence from Streptococcus pneumoniae (spSrtA). While some of our chimeric enzymes mimic the activity and selectivity of the wild-type protein from which the loop sequence is derived (e.g., that of saSrtA), others result in chimeric spSrtA enzymes able to accommodate a range of residues in the final position of the substrate motif (LPXT $\underline{X})$. Using mutagenesis, structural, and sequence analyses, we identify three interactions facilitated by $\beta 7-\beta 8$ loop residues that appear to be broadly characteristic of Class A sortase enzymes. These studies provide the foundation for a deeper understanding of sortase target selectivity and can expand the sortase toolbox for future SML applications.
\end{abstract}


Piper and Struyvenberg et al.

\section{Introduction}

Sortases are cysteine transpeptidase enzymes that gram-positive bacteria use to covalently attach proteins to their cell wall for various functions, including to assemble pili or display virulence factors (1-3). There are 6 recognized classes of sortase enzymes (classes A-F), with in vivo roles ranging from general purpose or "housekeeping" functions (classes A and E), to more specific roles such as the construction of the bacterial pilus (Class C) $(1,4)$. These enzymes recognize a cell wall sorting signal (CWSS) on the outer membrane of gram-positive bacteria (1, 5). For Class A sortases, the CWSS is the sequence LPXTG $(1,5)$. Using previously published numbering $(\mathrm{L}=\mathrm{P} 4, \mathrm{P}=\mathrm{P} 3, \mathrm{X}=\mathrm{P} 2, \mathrm{~T}=\mathrm{P} 1$, and $\mathrm{G}=\mathrm{P} 1$ ') $, \mathrm{P} 4, \mathrm{P} 3$ and/or $\mathrm{P} 1$ ' of this motif vary amongst different classes (5). Following target recognition, a His-Cys-Arg catalytic triad facilitates a ligation reaction whereby the CWSS is cleaved between threonine and glycine residues, followed by resolution of an acyl-enzyme intermediate via nucleophilic attack by an incoming amino group that results in formation of a new peptide bond $(1,3,5,6)$.

The ability to cleave a signal sequence and subsequently ligate a second component (typically a protein or synthetic peptide derivative) via a covalent bond make sortases an attractive tool for protein engineering efforts, commonly called sortase-mediated ligation (SML) or sortagging (3). Sortase A from Staphylococcus aureus (saSrtA) was the first of these enzymes discovered and continues to see widespread use for in vitro SML experiments (Fig. 1A-B) $(1,7)$. Recent years have seen continuous improvements in SML technology, including strategies for limiting the reversibility of the ligation reaction, and the development of saSrtA variants with dramatically improved catalytic efficiency $(3,8)$. However, in the majority of cases SML remains restricted to the pentapeptide LPXTG motif, which limits its utility as a protein engineering tool $(9,10)$. A relaxed recognition motif could potentially allow scientists to target a larger number of protein targets that do not contain an endogenous LPXTG sequence (11). 
Piper and Struyvenberg et al.

Previous mutagenesis and structural studies of various sortases provide a wealth of knowledge about initial ligand recognition and subsequent cleavage (thioesterification), as well as nucleophile recognition and mechanistic details of peptide ligation (transpeptidation) $(1,2,9)$. Specifically, the catalytic residues of all native sortases identified to date are (using saSrtA numbering unless specified otherwise): His120 (general acid/base), Cys184 (nucleophile, acyl-enzyme intermediate), and Arg197 (transition state stabilization) (Fig. 1B) (1, 9). Additionally, directed evolution studies have identified mutations (P94R/D160N/D165A/K190E/K196T) that are able to boost the catalytic efficiency of saSrtA by 120-fold (8). Of these 5 mutations, several are located in two of the three structurally conserved loops in Class A sortases: those between the $\beta 4, \beta 5$ strands ( $\beta 4-\beta 5$ loop), the $\beta 6, \beta 7$ strands ( $\beta 6-\beta 7$ loop, where D165A occurs), and the $\beta 7, \beta 8$ strands ( $\beta 7-\beta 8$ loop, where K190E and K196T are located). Notably, while the increase in enzyme activity afforded by these mutations included a 3.6-fold increase in $\mathrm{k}_{\text {cat }}$, the effect was dominated by a 33-fold decrease in $\mathrm{K}_{\mathrm{M}}$, suggesting these loop residues may be important in CWSS recognition (8).

Additional evidence for the role of loop residues has been obtained from more targeted directed evolution and mutagenesis studies. For example, it has been demonstrated that the $\beta 6-\beta 7$ loop of saSrtA directly confers specificity at P4 of the recognition motif (LPXTG), and residues other than leucine (L) can be accommodated using sortases with mutations in the $\beta 6-\beta 7$ loop. (1214). Indeed, substitution of the $\beta 6-\beta 7$ loop residues from saSrtB into the saSrtA enzyme alters substrate recognition to that of a sortase B protein (NPQTN) (15). Turning to the $\beta 7-\beta 8$ loop, the NMR structure of saSrtA covalently bound to a modified LPAT* peptide mimetic revealed a non-covalent interaction between W194 in saSrtA and the Thr residue in P1 (LPXTG) $(16,17)$. Mutation of W194 in saSrtA decreased the reaction rate, although it was not essential to catalysis 
Piper and Struyvenberg et al.

(17). Taken together, these past studies reveal that sequence variation within sortase loops directly affects both activity and selectivity for target ligands. Furthermore, conservation of the closed eight-stranded $\beta$-barrel core in all sortase A-F structures that have been reported to date suggests that these principles may apply to non-Class A sortases as well. (Figs. 1A, C) (2).

In this work we specifically look at natural sequence variation in the $\beta 7-\beta 8$ loop of Class A sortases, using Streptococcus pneumoniae SrtA as a model system. The $\beta 7-\beta 8$ loop was initially identified using principal component analysis as a region of notable variability in Class A (and other) sortases. We find that the $\beta 7-\beta 8$ loop sequence dramatically affects both overall enzyme activity, as well as selectivity at P1' of the CWSS. Our data is consistent with a recent publication that investigated the grafting of $\beta 7-\beta 8$ loop sequences from saSrtA and Bacillus anthracis SrtA into Streptococcus pyogenes SrtA (18). This work also suggested that W194 (saSrtA numbering) may play a role in the substrate recognition of the reported chimeras (18). Here, we have profiled the substrate preferences of over a dozen loop chimeras and single- or double-mutants targeting the $\beta 7-\beta 8$ loop. While we also observe a role for W194 in substrate recognition, our data suggests that it is unique to saSrtA and not broadly applicable to describe $\beta 7-\beta 8$ loop-mediated Class A sortase function. Indeed, the combination of functional enzyme assays and analysis of reported sortase structures in the present work suggests three different $\beta 7-\beta 8$ loop-mediated interactions that affect selectivity and activity. 
Piper and Struyvenberg et al.

\section{Results}

Principal component analysis (PCA) of bacterial sortases

In order to gain a better understanding of global sequence patterns in the sortase superfamily, we used principal component analysis (PCA) to group and analyze 39,188 sortase sequences from all classes (Experimental Procedures). Briefly, we downloaded all sequences annotated as "sortase" from UniProt and aligned then by MAFFT, followed by PCA $(19,20)$. The amino acids in each sequence were then classified by 5 parameters: hydrophobicity, disorder propensity, molecular weight, charge, and occupancy (defined as a binary value, where $1=$ amino acid and $0=$ insertion or deletion (indel) at this position) $(21,22)$. PCA was then performed on the resulting $39,188 * 5$ dimensional data. This data was projected onto 3 principal components where $42.7 \%$ of the total variance is described (Figs. S1A). Hierarchical clustering of the sortase superfamily was achieved by using the first two components of the singular value decomposition (SVD) matrix. The projected points were then partitioned into 2 clusters by Gaussian mixture modeling. This process is performed recursively on the dataset until each cluster reaches a minimum size or the Gaussian mixture modeling process fails to identify two distinct gaussians (23). The resulting tree from this process can accurately distinguish the known sortase classes (Fig. 2A). We also plotted our PCA using the top three principle components (Fig. S1B). For visualization, we ran PCA on a subset of the data, including 9,427 sequences that were filtered for low numbers indels and manually verified (Fig. 2B).

This analysis verified previous classifications of sortases based on sequence alignment, network, and phylogenetic tree analyses $(4,24,25)$. For example, principal component 1 (PC1) separates the sortase F proteins from the rest of the superfamily and PC2 captures the separation between sortase B and the other sortase families, as well as sortase E and sortase A. These analyses 
Piper and Struyvenberg et al.

allowed us to identify the regions of highest variability within each class based on the parameters defined above. We plotted our data onto previously determined sortase A structures by linearizing the distance from the centroid for each position in the multiple sequence alignment (Fig. S1C). Unsurprisingly, we found that secondary structure elements are highly conserved, including the "sortase fold" $\beta$-barrel core and class-specific $\alpha$-helices (Figs. 2C-D and S1D). Additionally, PCA revealed that the highest degree of variability occurs in structurally conserved loops adjacent to the substrate recognition pocket (Figs. 2C-D and S1D).

Given that the $\beta 6-\beta 7$ loop has been shown to be intimately involved in sortase substrate recognition, we were intrigued that PCA revealed similar levels of variability in the $\beta 4-\beta 5$ and $\beta 7-\beta 8$ loops (15). In the case of $\beta 7-\beta 8$, we were also motivated by previously reported mutations in the $\beta 7-\beta 8$ loop of saSrtA that have been shown to dramatically modulate sortase reaction rates $(8,17,26)$. Therefore, we sought to further explore how the $\beta 7-\beta 8$ loop affects the activity and substrate specificity of a sortase with narrow substrate tolerance ( $a$ SrtA) versus one that is more promiscuous (spSrtA).

Loop-swapped $\beta 7-\beta 8$ variants reveal differences in position P1' selectivity for $S$. aureus and S. pneumoniae SrtA enzymes

In our previous work, we found that while saSrtA is specific for a Gly residue at P1' (LPXTG $)$ of the substrate motif, SrtA from Streptococcus pneumoniae (spSrtA) recognizes over 10 of the 20 amino acids at this position in a 24-hr end point assay (27). To determine whether the $\beta 7-\beta 8$ loop played a role in these differing substrate preferences, we began by engineering two loop-swapped variants: saSrt $_{\text {pneumoniae }}$ (which contains the $\beta 7-\beta 8$ loop residues from spSrtA (CEDLAATER , where the catalytic cysteine and arginine are underlined), and spSrtA $\mathrm{Aureus}_{\text {(with }}$ 
Piper and Struyvenberg et al.

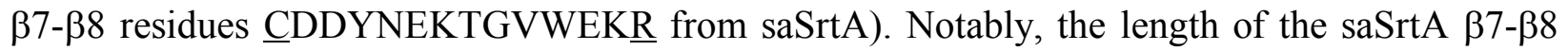
loop contains an additional five residues, as compared to the spSrtA $\beta 7-\beta 8$ loop. The saSrtA loop also uniquely contains W194, which is known to directly contact the P1 threonine of the LPXTG motif (16). In addition, while both loops are predicted to have an overall net negative charge at physiological $\mathrm{pH}$, the saSrtA loop contains two positively charged lysine residues that are not present in spSrtA. Both chimeric sortases were expressed and purified from E. coli, and were isolated as soluble, monomeric enzymes as described previously and in the Experimental Procedures (Fig. S2A) (27). The secondary structure content for all variants was consistent with the respective wild-type protein, as measured by circular dichroism and described in the Experimental Procedures (Fig. S2B).

To monitor enzymatic activity and selectivity of the saSrt $\mathrm{A}_{\text {pneumoniae}}, \mathrm{spSrt}_{\text {aureus, }}$ and their wild-type counterpart proteins, we utilized well-established FRET quencher probes consisting of different substrate motifs flanked by a 2-aminobenzoyl fluorophore (Abz) and a 2,4-dinitrophenyl quencher (Dnp) $(17,28,29)$. Probes containing three substrate variants were initially prepared (Abz-LPATAG-K(Dnp), Abz-LPATGG-K(Dnp), Abz-LPATSG-K(Dnp), varying only at P1' in bold) and used to test the relative activity of our wild-type and chimeric enzymes (Table S2). For simplicity, we have hereafter omitted the Abz, K(Dnp), and C-terminal glycine from peptide descriptions. For comparing enzyme activity, a standard $2 \mathrm{~h}$ reaction time was utilized, and an excess of $\mathrm{H}_{2} \mathrm{NOH}$ was included to resolve the acyl enzyme intermediates. For consistency, all reactions were also conducted in the presence of $\mathrm{Ca}^{2+}$, which is a required co-factor for saSrtA. Reaction endpoint (indicated by the increase in Abz fluorescence) for all enzyme/substrate pairings was then expressed relative to averaged benchmark reactions of wild-type saSrtA with the standard LPATG substrate (Figs. 3A, S3A). This benchmark reaction was consistently found to give $\sim 84 \%$ 
Piper and Struyvenberg et al.

conversion to the expected transacylation products when independently monitored via RP-HPLC

(Fig. S3B).

Based on our previous results, we predicted that spSrtA would show activity for all three peptides, while saSrtA would be selective for LPATG (27). Consistent with this prediction, our results confirmed that spSrtA was equally capable of processing all three substrates, whereas saSrtA was restricted to LPATG (Fig. 3A). Our assay also revealed a marked reduction in spSrtA activity versus saSrtA, which was not captured in our previous study, likely due to the extended reaction time (24 h) used in that work (27). With respect to the chimeric enzymes, our results clearly showed that the sequence of the $\beta 7-\beta 8$ loop was a major determinant of activity and specificity. Specifically, the saSrtA $\mathrm{A}_{\text {pneumoniae }}$ protein was completely inactive while $\mathrm{spSrt} \mathrm{A}_{\text {aureus }}$ functionally mimicked the narrow substrate preference of the wild-type saSrtA enzyme (Fig. 3A). This result is consistent with recently published data (18). To verify that our sortases were cleaving substrates at the expected site, reactions exhibiting a normalized fluorescence value of 0.2 were independently monitored by RP-HPLC and LC-MS, which confirmed cleavage between P1 and P1' (Figs. 3B, S3B-F). Notably, reactions for HPLC and LC-MS characterization were conducted in the presence and absence of $\mathrm{Ca}^{2+}$, which demonstrated that this cofactor was not required for the activity of spSrtA and spSrtA $\mathrm{A}_{\text {aureus. }}$

Continuing on with the $\mathrm{SpSrtA}_{\text {aureus }}$ chimera, we next wanted to determine if the Trp residue derived from the saSrtA loop played a significant role in enzyme activity. In wild-type saSrtA, the W194 residue (using saSrtA numbering) is known to affect enzyme activity of saSrtA via direct interactions with the threonine of the LPXTG motif $(16,17)$. We therefore expressed and purified the corresponding "W194T" mutant of spSrtA $\mathrm{Aureus}_{\text {and }}$ and tested this variant with A-, G-, and Scontaining peptides in our assay. Indeed, our W194T spSrtA $\mathrm{A}_{\text {aureus }}$ protein exhibited a 59\% 
Piper and Struyvenberg et al.

reduction in reaction progress for LPATG, while retaining its selectivity for Gly-containing peptides (Fig. 3A, Table S1). This result suggests that W194 likely interacts with the peptide substrate in a similar manner as previously described, despite the spSrtA scaffold (16).

Variability in position P1' selectivity and transpeptidase activity in S. pneumoniae SrtA $\beta 7-\beta 8$ variants

In addition to the profound shift in substrate scope observed for spSrtA $\mathrm{A}_{\text {aureus, }}$, we were also intrigued that the overall reactivity of this chimera for LPATG was comparable to that seen with wild-type saSrtA. This stood in sharp contrast to the reaction of LPATG with wild-type spSrtA, where reaction progress was nearly two-thirds lower within the $2 \mathrm{~h}$ reaction time of our assay (Fig. 3A). Based on this, we wondered if similar gains in reactivity for substrates other than LPATG could be achieved by substituting in residues from additional SrtA proteins $(27,28)$. To test this, we created an additional 6 spSrtA variants containing loop residues from SrtA proteins that we had evaluated previously (27). These chimeras included the $\beta 7-\beta 8$ loop residues from Bacillus

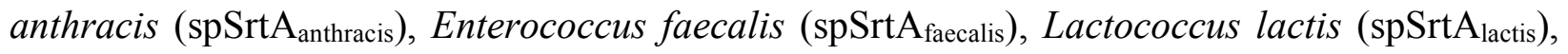

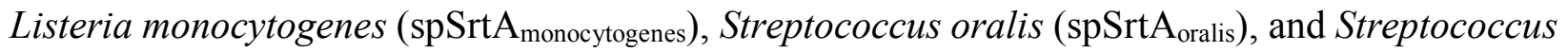
suis $\left(\right.$ spSrtA $\left._{\text {suis }}\right)$ (Fig. 4A) (27). To avoid confusion in the numbering of loops with variable lengths, we will hereafter refer to the N-terminal positions of each $\beta 7-\beta 8$ loop by numbering with respect to the catalytic Cys $\left(\beta 7-\beta 8^{+1}, \beta 7-\beta 8^{+2}\right.$, etc. $)$ that precedes the loop, whereas the C-terminal loop residue will be numbered relative to the catalytic $\operatorname{Arg}\left(\beta 7-\beta 8^{-1}\right)$ (Fig. 4A).

The 6 chimeric proteins were expressed and purified using the same protocol as spSrtA $_{\text {aureus, }}$ and as described in the Experimental Procedures. The purity of all proteins was validated by SDS-PAGE, and size exclusion chromatography was consistent with the isolated 
Piper and Struyvenberg et al.

proteins being predominantly monomeric (Fig. S2A). In addition, all variants contained similar secondary structure content as compared to the respective wild-type protein, as measured by circular dichroism (Fig. S4A). With the new chimeras in hand, we conducted an initial evaluation of relative activity using the LPATG, LPATA, and LPATS substrates described above. While the majority of constructs exhibited significant reactivity across all three substrates, the spSrt $\mathrm{A}_{\text {anthracis }}$ and $\mathrm{spSrt}_{\mathrm{A} o n o c y t o g e n e s}$ proved to be inactive (Fig. S4B). For the remaining enzymes, the spSrt $\mathrm{A}_{\text {oralis }}$ protein behaved similarly to wild-type spSrtA, while spSrtA $\mathrm{A}_{\text {faecalis, }}, \mathrm{sprrt}_{\text {lactis, }}$, and $\mathrm{spSrtA}_{\text {suis }}$ showed improved performance for A-, G-, and S-containing substrates (Figs. 4B-C, S4B). This was particularly interesting in the case of $\operatorname{spSrtA}_{\text {faecalis }}$ given that the wild-type SrtA enzyme from E. faecalis was previously shown to have poor reactivity for the same test substrates despite the use of higher enzyme loading and considerably longer reaction times (27).

Based on initial experiments with the A-, G-, and S-containing peptides, we next wanted to expand our peptide pool in order to assess the relative reactivity of our active chimeric spSrtA variants for peptides containing all 20 amino acids at P1'. For comparison, a similar substrate profile was generated for wild-type spSrtA. As shown in Fig. 4B, within the $2 \mathrm{~h}$ time frame of our assay, the wild-type protein was rather selective in its substrate recognition, with reactivity limited to A-, G-, and S-containing peptides (Table S1). We note here that this somewhat limited substrate scope appears to differ from the more promiscuous behavior reported previously for spSrtA. We attribute this to the fact that longer reaction times $(24 \mathrm{~h})$ and higher enzyme loadings (5-fold higher than the loading used here) were utilized in this earlier work (27). Similar to wild-type spSrtA, the spSrtA $_{\text {oralis }}$ was limited to A-, G-, and S-containing peptides, albeit with slightly elevated reactivity in the case of LPATA and LPATG. Finally, we were intrigued to find that our $\operatorname{spSrtA}_{\text {faecalis, }}$ 
Piper and Struyvenberg et al.

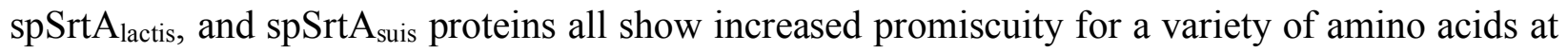
P1' in our assay (Fig. 4C, Table S1).

Overall, the $\operatorname{sSrtA}_{\text {faecalis, }}, \mathrm{sprt}_{\text {lactis}}$, and $\mathrm{spSrtA}_{\text {suis }}$ proteins showed the largest increase in activity and promiscuity for this library of peptides. The $\operatorname{sprtA}_{\text {faecalis }}$ and $\mathrm{spSrt} \mathrm{A}_{\text {lactis }}$ proteins each recognized 15 of the 20 amino acids at $\mathrm{P} 1$ ' with normalized fluorescence values of $\geq 0.05$, while spSrtA $_{\text {suis }}$ recognized 14 of the 20 (Table S1). We chose 0.05 as a cut-off value in order to compare with the peptide activities of the spSrtA protein, which shows normalized fluorescence values of - 0.02 to 0.02 for all non-G-, S-, or A-containing peptides, with the exception of LPATC (at 0.04 \pm 0.01 ) (Table S1). Furthermore, $\operatorname{spSrtA}_{\text {faecalis }}$ and $\operatorname{spSrtA}_{\text {suis }}$ exhibited $\sim 3$-fold higher reaction progress for the G-, S-, and A-containing peptides, as compared to spSrtA (Table S1).

As verification of the results of our fluorescence assay, we also characterized a subset of enzyme/substrate combinations using RP-HPLC and LC-MS. Focusing on spSrtA faecalis we repeated reactions that exhibited normalized fluorescence values of $\geq 0.1$ (LPATX, $\mathbf{X}=\mathrm{A}, \mathrm{F}, \mathrm{G}$, I, L, M, N, S, V, W, Y) (Fig. S4C). Reactions were conducted in the absence of $\mathrm{Ca}^{2+}$ to confirm that this cofactor was not required for activity. Successful substrate cleavage was observed in all cases, ranging from a high of $78 \%$ conversion in the case of LPATG, to only $6 \%$ conversion in the case of LPATW over 2 hours at temperature (Fig. S4C). Notably, the trends in relative substrate preferences observed by HPLC were consistent with those found in our original fluorescence assay (Fig. S5). Additionally, while LC-MS characterization confirmed that substrate cleavage was occurring between the $\mathrm{P} 1$ and $\mathrm{P} 1$ ' of all sequences, certain substrates (LPATX, $\mathbf{X}=\mathrm{W}, \mathrm{F}, \mathrm{L}$ ) containing bulky hydrophobic residues also produced alternate products arising from cleavage on the C-terminal side of P1'. In the case of LPATL, this alternate cleavage product was actually the major species obtained following reaction with $\operatorname{spSrtA}_{\text {faecalis. }}$ We note here that this capacity for 
Piper and Struyvenberg et al.

alternate cleavage has been reported previously for wild-type spSrtA, and thus appears to be maintained in the spSrtA faecalis chimera (27).

Variability in position P1' selectivity and ligase activity in S. pneumoniae SrtA $\beta 7$ - $\beta 8$ variants

As a final assessment of the reactivity of the $\operatorname{spSrt}_{\text {faecalis }}$ chimera, we next evaluated its ability to ligate amino acid nucleophiles in place of the $\mathrm{H}_{2} \mathrm{NOH}$ that was utilized in our fluorescence assay. For a series of test substrates (LPATX, $\mathbf{X}=\mathrm{A}, \mathrm{S}, \mathrm{V}), \operatorname{spSrtA} \mathrm{A}_{\text {faecalis }}$ was able to successfully ligate the corresponding free amino acid carboxamides $\left(\mathbf{X}-\mathrm{NH}_{2}=\mathrm{A}-\mathrm{NH}_{2}, \mathrm{~S}-\mathrm{NH}_{2}\right.$, V-NH${ }_{2}$ ) with very good efficiency (Fig. 5). As expected from our fluorescence assay results, reaction progress with LPATV was slower than that observed for LPATA and LPATS. Specifically, reactions with LPATV required $8 \mathrm{~h}$ at room temperature to consume $85 \%$ of the initial peptide substrate, whereas reactions with LPATA/S exhibited $>95 \%$ substrate conversion within 3 h. Importantly, the desired LPATX-NH 2 species was the major ligation product in all reactions as determined by LC-MS (Fig. 5, Table S2). Trace levels of substrate hydrolysis were also observed via LC-MS, however the ratio of successful ligation to hydrolysis was 15:1 or better as estimated from mass spectral peak intensities. In reactions involving LPATV, we also detected low levels of substrate cleavage on the C-terminal side of the P1' valine residue. The extent of this alternate cleavage pathway was minimal, accounting for only $\sim 4 \%$ of the substrate cleavage events based on comparisons of HPLC peak areas for G-K(Dnp) and VG-K(Dnp) (Fig. 5). Interestingly, LC-MS characterization of these same reactions involving LPATV, $\mathbf{V}-\mathrm{NH}_{2}$, and $\operatorname{spSrt} \mathrm{A}_{\text {faecalis }}$ failed to show clear evidence for the formation of ligation or hydrolysis products derived from the alternate cleavage pathway, potentially due to their low levels in solution. 
Piper and Struyvenberg et al.

For comparison, we also performed the same set of test ligations with wild-type spSrtA. In all cases, reaction progress was significantly reduced as compared to $\operatorname{spSrtA}_{\text {faecalis }}$ (Fig. 5). In particular, spSrtA exhibited minimal product formation with the LPATV system, representing a 10 -fold reduction in reaction progress relative to $\mathrm{spSrtA}_{\text {faecalis }}$ for this atypical sortase substrate motif. Building from this result, an initial attempt to utilize the LPATV sequence as a handle for site-specific protein modification was made by installing this motif at the C-terminus of a full-size protein target. However, this protein substrate proved to be unreactive in the presence of both $\operatorname{spSrtA}_{\text {faecalis }}$ and wild type spSrtA (data not shown).

\section{Stereochemical basis of $\beta 7-\beta 8$ variant selectivity and activity}

In order to gain a stereochemical understanding of our biochemical results, we first analyzed available structures of Class A sortases in the Protein Data Bank. To our knowledge, the 3D structure of an active, monomeric form of spSrtA has yet to be reported. Available crystal structures of the domain-swapped dimer show that the $\beta 7-\beta 8$ loop is located at, and participates in, the dimer interface (PDB codes 4O8L, 4O8T, and 5DV0). Therefore, we chose to broaden our search to non-spSrtA structures, and in doing so we identified 3 putative $\beta 7-\beta 8$ loop-mediated interactions in Class A sortases:

(1) A Thr-mediated intra-loop hydrogen bond. We initially observed an intra-loop hydrogen bond formed in the $\beta 7$ - $\beta 8$ loops of several Class A sortases (Fig. 6A). This Thr-mediated hydrogen bond is evident in SrtA proteins from $S$. aureus (PDB code 2KID), S. pyogenes (3FN5), S. mutans (4TQX), and L. monocytogenes SrtA (5HU4), among others not shown (Fig. 6A). We predict that in spSrtA, this hydrogen bond will be formed between the side chains of residues $\beta 7-\beta 8^{+2}$ D209 and $\beta 7-\beta 8^{+6}$ T213. Indeed, we see this interaction in a homology model of the 
Piper and Struyvenberg et al.

monomeric spSrtA protein generated using SwissModel (Fig. S6A). For this model, the Streptococcus pyogenes SrtA structure (PDB code 3FN5) was used as a template because the crystallized form of this enzyme (S. pyogenes SrtA residues S81-T249) has 63\% sequence identity with spSrtA (30-32). An alignment of our spSrtA model with 3FN5 revealed an overall RMSD of $0.083 \AA$ over 567 main chain atoms. We further validated our model using structural alignments with a monomer extracted from the domain-swapped dimer structure (RMSD of $0.603 \AA$ over 483 main chain atoms), as well as other SrtA structures from Streptococcus species, including those from Streptococcus agalactiae and Streptococcus mutans (PDB codes 3RCC (RMSD of $0.773 \AA$ over 384 main chain atoms) and 4TQX (RMSD of $0.456 \AA$ over 530 main chain atoms), respectively) (Fig. S6B-C).

We hypothesize that this $\beta 7-\beta 8$ intra-loop hydrogen bond is important for reducing flexibility in the $\beta 7-\beta 8$ loop, which may be an important characteristic of certain sortases prior to substrate recognition. This is apparent in saSrtA (PDB code 2KID), where NMR structures reveal that the $\beta 7-\beta 8$ loop is ordered in both the unbound and bound states (Fig. S7A). In contrast, previously published NMR structures of the B. anthracis SrtA (baSrtA) protein (PDB codes $2 \mathrm{KW} 8$ and 2RUI), which does not contain a $\beta 7-\beta 8$ loop Thr, point to a distinct mechanism. These structures show that a unique $\mathrm{N}$-terminal appendage in baSrtA regulates active site accessibility and that the $\beta 7-\beta 8$ loop transitions from a disordered-to-ordered state upon substrate binding (Fig. S7B) (33). In the ordered/bound state, there are $\beta 7-\beta 8$ intra-loop hydrogen bonds, specifically between two Ser residues with the peptide backbone and/or D192, but overall, these interactions appear to be distinct from the Thr-mediated hydrogen bonding discussed above. Our substrate profiling results, which showed that the $\mathrm{spSrt}_{\text {anthracis }}$ protein was inactive, are consistent with the 
Piper and Struyvenberg et al.

idea that spSrtA and saSrtA enzymes have a different substrate-recognition mechanism than baSrtA (Fig. S4B).

(2) A non-covalent interaction between the $\beta 7-\beta 8^{-1}$ residue and the $\beta 6^{-2}$ residue. We observe interactions between the $\beta 7-\beta 8^{-1}$ and $\beta 6^{-2}$ residues in multiple Class A sortase structures (Fig. 6B). For example, K196 of saSrtA interacts with the $\beta 6^{-2}$ D160 in several of the states of the NMR structure, PDB code 2KID (Fig. 6B) (16). We also see a reasonable electrostatic interaction distance for the $\beta 7-\beta 8^{-1}$ and $\beta 6^{-2}$ residues (K195 and E165, respectively) in several of the NMR states for B. anthracis SrtA (baSrtA) (PDB code 2RUI), as well as in the domain-swapped dimer structure of spSrtA, which shows the E214 $\beta 7-\beta 8$ loop residue of one protomer interacting with R184 (Fig. 6B). Interestingly, the nature of this interaction can change, e.g., in Actinomyces oris SrtA (aoSrtA) where both residues are hydrophobic leucine residues (L186 and L228 for the $\beta 6^{-2}$ and $\beta 7-\beta 8^{-1}$ residues, respectively) (Fig. 6B).

Notably, our spSrtA homology model suggests that in spSrtA, the $\beta 7-\beta 8^{+1}$ E208 may also interact with the $\beta 6^{-2} \mathrm{R} 184$, with a distance between a guanidinium nitrogen atom of R184 and a side chain carboxylate oxygen atom on E208 equal to $2.7 \AA$ (Fig. S6A). Because this residue is a glycine in the more active spSrt $\mathrm{A}_{\text {faecalis }}$ enzyme, we wanted to see if this $\beta 7-\beta 8^{+1}$ Glu contributes to the relatively low reactivity of spSrtA by mutating the Gly in this position to Glu in our $\mathrm{spSrtA}_{\text {faecalis }}$ protein, or G145E (using E. faecalis SrtA numbering). We expressed and purified $\mathrm{G}_{145 \mathrm{E} \text { spSrtA }}$ faecalis and tested the protein with our A-, G-, and S-containing peptides. Indeed, we saw a $21-49 \%$ reduction in the activity for these three peptides relative to our initial $\mathrm{spSrt}_{\text {faecalis }}$ variant, suggesting this residue may play a role in the activity of the enzyme (Fig. 6C, Table S1). The G145E spSrt $A_{\text {faecalis }}$ protein is the only variant that contains less secondary structure content than the wild-type protein; therefore, we cannot rule out protein misfolding in these data (Fig. 
Piper and Struyvenberg et al.

S4A). However, considering the $\beta 7-\beta 8$ loops of spSrtA and E. faecalis SrtA differ at only the $\beta 7-\beta 8^{+1}$ (E in spSrtA, G in E. faecalis SrtA), $\beta 7-\beta 8^{+4}$ (A versus Q, respectively), and $\beta 7-\beta 8^{-1}$ (E versus T, respectively) positions (Fig. 4A), we hypothesize that this potential interaction at the $\beta 7-\beta 8^{+1}$ position does negatively affect enzyme activity in the spSrtA and G145E spSrtA $\mathrm{A}_{\text {faecalis }}$ proteins.

(3) A hydrophobic interaction between the $\beta 7-\beta 8^{+3}$ residue and the $\beta 4-\beta 5$ loop (positions +2 or +3 from the catalytic His residue, or $\left.\beta 4-\beta 5^{+2} / \beta 4-\beta 5^{+3}\right)$. In analyses of the previously published baSrtA structures, the authors of this work mention that the $\beta 4-\beta 5^{+2} / \beta 4-\beta 5^{+3}$ positions (for baSrtA, these are $\beta 4-\beta 5^{+2}$ M128 and $\beta 4-\beta 5^{+3}$ S129), play a role in stabilizing a hydrophobic residue, the $\beta 7-\beta 8^{+3}$ V190, upon ligand binding (Fig. 6D) (33). Although we do not see a similar interaction in the bound saSrtA structure, we do see a similar movement of the $\beta 7-\beta 8^{+3}$ residue towards these $\beta 4-\beta 5$ loop residues (Fig. 6D). The L. monocytogenes SrtA (or lmSrtA) structure (PDB ID 5HU4) also shows this interaction, $\beta 4-\beta 5^{+2}$ M129 and $\beta 4-\beta 5^{+3}$ R130 with P191 in the $\beta 7-\beta 8^{+3}$ position, in the unbound state (Fig. 6D). Finally, our spSrtA model suggests a potential interaction between the $\beta 4-\beta 5^{+2}$ I63 and $\beta 4-\beta 5^{+3}$ F64 residues with $\beta 7-\beta 8^{+3}$ L210 (Fig. 6D).

Mutagenic investigation of the contribution of $\beta 7-\beta 8$ loop residues

In order to investigate these hypothesized interactions, we tested a number of $\beta 7-\beta 8$ mutants in spSrtA. Specifically, we expressed and purified the following mutant spSrtA proteins, as described in the Experimental Procedures, and characterized them using size exclusion chromatography and circular dichroism: E208G, L210P, T213A, D209T/T213D, and E214G (Figs. 6E, S4A and S7C). Our results are consistent with the proposed interactions. Specifically, 
Piper and Struyvenberg et al.

the T213A mutation, which disrupts a potential intra-loop hydrogen bond between the $\beta 7-\beta 8^{+2}$ D209 and $\beta 7-\beta 8^{+6}$ T213 reduces spSrtA activity by 54-73\% for G-, S-, and A-containing peptides (Fig. 6E, Table S1). When we attempt to reverse the hydrogen bond geometry with the D209T/T213D double mutant, we see no enzyme activity (Fig. 6E, Table S1). In contrast to an enhancement in activity when the intra-loop hydrogen bond is present, the $\beta 7-\beta 8^{-1}$ and $\beta 6^{-2}$ proposed interaction negatively impacts spSrtA activity. The E214G mutant spSrtA revealed an almost 3-fold increase in activity, as compared to its wild-type counterpart (Fig. 6E, Table S1). This is consistent with recent work where a triple mutant of $S$. pyogenes SrtA (E189H/V206I/E215A, where E215A is a mutation at the equivalent $\beta 7-\beta 8^{-1}$ position) resulted in a 6.6-fold enhanced catalytic efficiency (26). Notably, K196T in the catalytically enhanced pentamutant saSrtA protein is also located at the $\beta 7-\beta 8^{-1}$ position (8). We also see that the $\beta 7-\beta 8^{+1}$ Glu negatively affects enzyme activity, as we predicted based on our G145E spSrtA $\mathrm{A}_{\text {faecalis }}$ results; here, the E208G spSrtA protein has over 2-fold higher activity than the wild-type protein (Fig. 6E, Table S1). We predict that in spSrtA, both E208 and E214 can interact with $\beta 6^{-2}$ R184, a hypothesis that our spSrtA model supports (Fig. S6A). Finally, the L210P spSrtA enzyme shows similar activity to the T213A mutant (Fig. 6E, Table S1), supporting a positive role for the proposed $\beta 7-\beta 8^{+3}$ interaction with $\beta 4-\beta 5^{+2} / \beta 4-\beta 5^{+3}$, similar to that seen in baSrtA (33). This result may explain why our spSrtA monocytogenes protein is catalytically inactive, as the L. monocytogenes SrtA $\beta 7-\beta 8$ loop contains a Pro at this position (Figs. 4A, S4B).

We tabulated the residues at relevant positions for the wild-type Class A sortases in the 8 organisms we used for our spSrtA chimeras in Table 1. Without structural data, we cannot conclude that all 3 interactions are present in all of these proteins, although most appear to be conserved at the sequence level. There are two notable exceptions, the sequences of L. lactis and 
Piper and Struyvenberg et al.

S. suis SrtA proteins contain polar or charged residues at the $\beta 7-\beta 8^{-1}$ position (H213 and Q213, respectively), but hydrophobic residues at the $\beta 6^{-2}$ residue (V183 and I183, respectively), which is inconsistent with a predicted interaction (Table 1). Homology models of both (template: 4TQX for L. lactis SrtA and 3FN5 for $S$. suis SrtA) suggest that the $\beta 6-\beta 7^{+1}$ position in these proteins may be an interaction partner, although it is unclear (Figs. S7D-E). Although the other 8 SrtA sequences analyzed in Table 1 do contain $\beta 6-\beta 7^{+1}$ polar or charged residues (listed in the footnote of Table 1), no available SrtA structures show an interaction between this residue and the $\beta 7-\beta 8$ loop. Additional studies on these two proteins may elucidate whether or not this $\beta 7-\beta 8$ loop interaction is present and/or what, if any, effect there is on the activity and selectivity of the enzyme.

Sequence patterns in $\beta 7$ - $\beta 8$ loop sequences of Class $A$ sortases

Based on our structural observations using published structures and homology models, as well as our mutagenesis activity data, we identified 3 sequence motifs that appeared to be present in multiple Class A sortases. These include: (1) an intra-loop hydrogen-bonding pair, typically between the $\beta 7-\beta 8^{+1 /+2}$ loop and $\beta 7-\beta 8^{+6 /+7}$ positions, with the latter usually a Thr residue, (2) an interaction between the $\beta 7-\beta 8^{-1}$ and $\beta 6^{-2}$ positions, and (3) an interaction between the $\beta 7-\beta 8^{+3}$ with the $\beta 4-\beta 5^{+2}$ and $\beta 4-\beta 5^{+3}$ positions, usually of hydrophobic nature. In an effort to determine whether these motifs were more broadly present across the entire family of Class A sortases, we downloaded 387 Class A sortase sequences from the NCBI database. These were then aligned using MAFTT, and the $\beta 7-\beta 8$ loop sequences were manually extracted, using the catalytic Cys and Arg residues to define the boundaries of each (Table S3). Of those sequences, we analyzed a subset of 261 from the following genera: Bacillus (44 sequences), Enterococcus (68 sequences), 
Piper and Struyvenberg et al.

Lactobacillus (102 sequences), Listeria (13 sequences), Staphylococcus (24 sequences), and Streptococcus (10 sequences).

We created WebLogos with sequences from each genus that had the same loop length and discovered a number of sequence patterns consistent with our predictions (Figs. 7, S8). Specifically, almost all of the sequences contain a $\beta 7-\beta 8^{+1 /+2}$ Asp/Glu paired with a $\beta 7-\beta 8^{+6 /+7} \mathrm{Thr}$ residue, the potential stabilizing hydrogen bonding pattern described above (Figs. 7, S8). The two exceptions to this pattern are Lactobacillus sequences that are either 6 or 9 amino acids. In the 9 residue Lactobacillus sequences, however, there is a very strong prevalence of a $\beta 7-\beta 8^{+2} \mathrm{Ser} / \mathrm{Thr}$ with a $\beta 7-\beta 8^{+7}$ Glu, a residue pair which may also form a hydrogen bond. Consistent with this idea, in aoSrtA, there is an intra-loop hydrogen-bond between the $\beta 7-\beta 8^{+3} \mathrm{~S} 219$ and $\beta 7-\beta 8^{+11} \mathrm{D} 227$ residues of Actinomyces oris SrtA (aoSrtA) (PDB ID 5UTT) (Fig. S9A).

Second, of the 226 sequences that we included in our WebLogo analysis, only 32 do not contain a charged or polar residue in the $\beta 7-\beta 8^{-1}$ position immediately preceding the catalytic Arg, including 19 Lactobacillus (Gly or Phe) and 13 Bacillus sequences (Gly or Trp) (Table S3). If we analyze these outliers further, we see that most often, including in Lactobacillus pantheris, which contains a Phe at this position, as well as in at least 8 Lactobacillus strains with a Gly at the $\beta 7-\beta 8^{-1}$ position, we see Val, Phe, or Tyr residues at the $\beta 6^{-2}$ position, which is consistent with a favorable hydrophobic interaction between these residues (Fig. S9B). This interaction is seen in the aoSrtA structure, between residues $\beta 7-\beta 8^{-1}$ L228 and $\beta 6^{-2}$ residue L186, described previously (Fig. 6B).

Finally, our WebLogo analysis confirms that the large majority of our sequences contain a $\beta 7-\beta 8^{+3}$ hydrophobic (or proline) residue. In cases where this is not true (e.g., Lactobacillus ozensis, which has a $\beta 7-\beta 8^{+3}$ Thr residue) the $\beta 4-\beta 5^{+2}$ and $/$ or $\beta 4-\beta 5^{+3}$ residues are often also not strictly hydrophobic (e.g., for L. ozensis, the $\beta 4-\beta 5^{+3}$ is a Glu) (Fig. S9B). Taken together, the 
bioRxiv preprint doi: https://doi.org/10.1101/2021.03.27.437355; this version posted March 28, 2021. The copyright holder for this preprint (which was not certified by peer review) is the author/funder, who has granted bioRxiv a license to display the preprint in perpetuity. It is made available under aCC-BY-NC-ND 4.0 International license.

Piper and Struyvenberg et al.

three $\beta 7-\beta 8$ loop-mediated interactions described here appear to be broadly conserved in Class A sortases. 
Piper and Struyvenberg et al.

\section{Discussion}

Although target sequence recognition by $S$. aureus SrtA is rigidly selective for a P1' glycine, this is not true of all Class A sortases, such as those from Streptococcus pneumoniae and Streptococcus pyogenes $(10,30,34,35)$. Building from our previous work, in which spSrtA was found to accept peptides containing Gly-, Ala-, Ser- and other residues at P1', we have shown here that this broadened substrate scope can be attributed to the sequence of the $\beta 7-\beta 8$ loop (26). Moreover, variations in $\beta 7-\beta 8$ loop sequences can substantially impact overall enzyme activity, affording chimeric sortases that outperform their wild-type counterpart in vitro. Together with others, the present study implicates all of the variable loops in Class A sortases as being important determinants of enzyme function $(13,15,18,33)$.

With respect to structure, we propose three interactions that are facilitated by residues in the $\beta 7-\beta 8$ loop of spSrtA, characteristics which we suggest are broadly shared by Class A sortases. They are: (1) an intra-loop hydrogen bond that positively affects catalytic efficiency, typically mediated by a threonine residue at the $\beta 7-\beta 8^{+6}$ or $\beta 7-\beta 8^{+7}$ position, (2) an interaction that hinders enzyme activity between the $\beta 7-\beta 8^{-1}$ and $\beta 6^{-2}$ residues, and (3) a positive interaction between the $\beta 7-\beta 8^{+3}$ and $\beta 4-\beta 5^{+2} / \beta 4-\beta 5^{+3}$ residues, typically of hydrophobic nature. Notably, there appear to be other structural features in this structurally conserved loop that are unique to certain Class A sortases. These include the W194T residue of saSrtA, which specifically interacts with the P1 position of the CWSS (16). Others identified a disordered-to-ordered transition of the baSrtA $\beta 7-\beta 8$ loop, as well as regulation by an N-terminal appendage, although more research is needed to determine whether or not this is shared by other Class A sortases (33). Of the seven-residue $\beta 7-\beta 8$ loop in spSrtA, we did not specifically investigate the $\beta 7-\beta 8^{+4}$ or $\beta 7-\beta 8^{+5}$ positions, which are ${ }^{211} \mathrm{AA}^{212}$ in spSrtA. Other Streptococcus SrtA structures, including those from S. pyogenes 
Piper and Struyvenberg et al.

$\left({ }^{212} \mathrm{EA}^{213}\right)$, S. agalactiae $\left({ }^{188} \mathrm{EA}^{189}\right)$, and $S$. mutans $\left({ }^{209} \mathrm{GA}^{210}\right)$ show that these residues are interacting with solvent and often not well modeled in crystal structures (Fig. S9C). We reveal, however, that all other residues in the $\beta 7-\beta 8$ loop, e.g., the $\beta 7-\beta 8^{+1}$ E208 residue in spSrtA, can affect activity, suggesting that it would be interesting to look at positional effects in Class A sortases with variable length $\beta 7-\beta 8$ loops.

In addition to informing our fundamental understanding of sortase substrate recognition, this work also has implications for the continued development of sortase-mediated ligation (SML) as a protein engineering tool $(3,36)$. Through exchange of $\beta 7-\beta 8$ loop residues between Class A sortases, we have generated chimeras such as $\operatorname{spSrt}_{\text {faecalis }}$ and $\operatorname{spSrt} \mathrm{A}_{\text {lactis }}$ with measurable activity against peptides possessing 15 of the 20 amino acids at $\mathrm{P} 1 '$ '. With additional development, each of these sortase chimera/substrate combinations potentially offers a new handle for in vitro SML applications. While preliminary attempts here to modify a protein target displaying an LPATV sequence using spSrtA faecalis were unsuccessful, we consider it likely that optimization of the placement of the LPATV site may restore reactivity. This includes examination of the accessibility requirements for the LPATV sequence, and assessment of the impact of residues N- or C-terminal to the core LPATV motif. Similar factors are known to affect the success of SML reactions with the widely used saSrtA/LPXTG system (37-39), and may need to be evaluated for our chimeras.

If successful, the development of these new sortase/substrate pairs has exciting consequences for SML engineering efforts: (1) it increases options for dual-labeling single proteins or multiplexed labeling of multiple proteins in the same systems $(11,40,41)$, and (2) it may reduce the need to mutate naturally occurring protein sequences in order to render their termini compatible with SML. For example, using our previously published program, MotifAnalyzer, we found 190 
Piper and Struyvenberg et al.

instances of LPXTG in 189 unique proteins in the human proteome. However, if the P1' position is now flexible, this number becomes 3606 instances of LPXTX in 2930 unique proteins (42).

Finally, the three variable loops mentioned here $(\beta 4-\beta 5, \beta 6-\beta 7$, and $\beta 7-\beta 8)$ are conserved in all classes of sortases (Fig. 1C), and previous work determining and engineering sortase selectivity of different classes, e.g., sortase B, suggests similar roles for these loops in substrate recognition $(5,15,43)$. Developing a deeper understanding of how residues in these loops affect substrate selectivity in all sortase classes may enable dramatic expansion of the sortase "toolbox" (Fig. 8), potentially allowing the development of ligases that are tailored to the needs of specific protein targets while also limiting off-target effects $(5,11,13,14,27,34,44)$. In the over 20 years since saSrtA was discovered, the sortase superfamily has proven to be both a workhorse for protein engineering efforts, as well as an exciting system for future discoveries and insight into the stereochemistry and mechanisms of target recognition. 
Piper and Struyvenberg et al.

\section{Experimental Procedures}

Principal component analysis (PCA). Initial sequences were obtained from UniProt and an alignment was generated by MAFFT $(19,20)$. Initially, each sequence was given a score for the number of gaps present for each residue and the filtered alignment was realigned by MAFFT. Subsequent analysis included all sequences without taking gaps into consideration (Fig. 2 B versus Figs. 2A, S2C). The sortase multiple sequence alignment (MSA) was converted to a matrix representing the chemical properties of the amino acids, each amino acid was associated with 4 biochemical traits and a binary trait occupancy, as described. Each trait was normalized to the range from zero to one. In addition, gaps were given the average value of the matrix column with the exception of occupancy, so that they would not contribute to variance of the column. Gapped positions were given an occupancy score of zero (for the other chemical properties gapped positions received the average score). After translating the MSA, the resulting matrix was re-centered so that the matrix had a column-wise mean of zero. Principal component analysis was performed on the matrix by the singular value decomposition algorithm provided in the scikit learn Python package (45). Clustering was performed by a Gaussian mixture model provided in the scikit-learn Python package (45). Optimal cluster numbers were scored by Bayesian information criterion. Visualization was performed using a script written in Python with matplotlib.

Protein expression and purification. Wild-type spSrtA and saSrtA proteins were expressed and purified as previously described (27). All other constructs, including chimeric and mutant proteins, were purchased from Genscript in the pET28a $(+)$ vector. In general, protein expression and purification protocols were very similar to those previously described (27). Briefly, plasmids were 
Piper and Struyvenberg et al.

transformed into Escherichia coli BL21 (DE3) competent cells and grown in LB media, with protein induction at $\mathrm{OD}_{600} 0.6-0.8$ using $0.15 \mathrm{M}$ IPTG for $18-20 \mathrm{~h}$ at $18^{\circ} \mathrm{C}$.

Following cell harvest in lysis buffer $[0.05 \mathrm{M}$ Tris $\mathrm{pH} 7.5,0.15 \mathrm{M} \mathrm{NaCl}, 0.0005 \mathrm{M}$ ethylenediaminetetraacetic acid (EDTA)], the protein was purified using a $5 \mathrm{~mL}$ HisTrap HP column (GE Life Sciences, now Cytiva), using wash [0.05 M Tris pH 7.5, 0.15 M NaCl, 0.02 M Imidazole $\mathrm{pH} 7.5,0.001 \mathrm{M}$ TCEP] and elution [wash buffer, with 0.3 M Imidazole pH 7.5] buffers. Size exclusion chromatography (SEC) was conducted using a HiLoad 16/600 Superdex 75 column (GE Life Sciences, now Cytiva) in SEC running buffer [0.5 M Tris pH 7.5, 0.15 M NaCl, 0.001 M TCEP]. Purified protein corresponding to the monomeric peak was concentrated using an Amicon Ultra-15 Centrifugal Filter Unit (10,000 NWML) and analyzed by SDS-PAGE and analytical SEC (Figs. S2, S7). Protein not immediately used was flash frozen in SEC running buffer and stored at $-80^{\circ} \mathrm{C}$.

Peptide synthesis. Detailed synthetic procedures are provided in the Supplemental Data. Briefly, all peptides were synthesized via manual Fmoc solid phase peptide synthesis (SPPS). Peptides were synthesized either individually or in tandem using Fmoc Rink amide MBHA resin or Synphase lantern solid supports. All other materials, including suitably protected Fmoc amino acids, and reagents for coupling, deprotection, and resin cleavage were obtained from commercial sources and used without further purification. All peptides were purified using RP-HPLC and their identities were confirmed via ESI-MS. Prior to use in sortase-catalyzed transacylation reactions, each purified peptide was prepared as a concentrated stock solution in DMSO and/or $\mathrm{H}_{2} \mathrm{O}$ (see Supplemental Data for details). 
Piper and Struyvenberg et al.

Fluorescence Assay for Sortase Activity. Reactions were performed in a Costar round-bottom, black 96-well plate at a $100 \mu \mathrm{L}$ reaction volume under the following conditions: $5 \mu \mathrm{M}$ sortase, 50 $\mu \mathrm{M}$ peptide substrate, and $5 \mathrm{mM}$ hydroxylamine nucleophile. All reactions contained $10 \%(v / v)$ 10x sortase reaction buffer (500 mM Tris $\mathrm{pH} 7.5,1500 \mathrm{mM} \mathrm{NaCl}$, and $\left.100 \mathrm{mM} \mathrm{CaCl}_{2}\right)$. Reactions also contained residual DMSO from the peptide stock solutions $(0.5-1.5 \%(v / v)$, with the exception of the Phe- and Val-containing peptides at 5\%). The peptides containing phenylalanine or valine required 5\% $(v / v)$ DMSO for solubility under the reaction conditions. $1 \mathrm{mM}$ TCEP was also included in reactions utilizing the Abz-LPATCG-K(Dnp) substrate. Reactions were initiated by the addition of the sortase enzyme, which were prepared as 10x stock solutions in $50 \mathrm{mM}$ Tris $\mathrm{pH}$ 7.5, $150 \mathrm{mM} \mathrm{NaCl}$, and $1 \mathrm{mM}$ TCEP. Microplates were analyzed using a Biotek Synergy H1 plate reader. The fluorescence intensity of each well was measured at 2-min time intervals over a 2-hr period at room temperature $\left(\lambda_{\mathrm{ex}}=320 \mathrm{~nm}, \lambda_{\mathrm{em}}=420 \mathrm{~nm}\right.$, and detector gain $\left.=75\right)$. All reactions were performed in triplicate (Table S1). For each substrate sequence, the background fluorescence of the intact peptide in the absence of enzyme was subtracted from the observed experimental data. Background-corrected fluorescence data was then normalized to the fluorescence intensity of a benchmark reaction between wild-type saSrtA and Abz-LPATGG-K(Dnp) (Fig. S3A).

HPLC and LC-MS Characterization of Sortase-Catalyzed Reactions. Select pairings of sortase enzyme $\left(5 \mu \mathrm{M}\right.$, or $10 \mu \mathrm{M}$ for the $\mathrm{X}-\mathrm{NH}_{2}$ reactions), substrate $(50 \mu \mathrm{M})$, and nucleophile (5 mM $\mathrm{H}_{2} \mathrm{NOH}$ or $\mathrm{X}-\mathrm{NH}_{2}$ ) were repeated in the presence or absence of $\mathrm{Ca}^{2+}$ under reaction conditions that were otherwise identical to those described above for the fluorescence assay. These reactions were then analyzed using a Dionex Ultimate 3000 HPLC system interfaced with an Advion CMS $\operatorname{expression}^{\mathrm{L}}$ mass spectrometer. Separations were achieved with a Phenomenex Kinetix® $2.6 \mu \mathrm{M}$ 
Piper and Struyvenberg et al.

C18 $100 \AA$ Å column $(100 \times 2.1 \mathrm{~mm})$ [aqueous $\left(95 \% \mathrm{H}_{2} \mathrm{O}, 5 \% \mathrm{MeCN}, 0.1 \%\right.$ formic acid $) / \mathrm{MeCN}$ $(0.1 \%$ formic acid $)$ mobile phase at $0.3 \mathrm{~mL} / \mathrm{min}$, method: hold $10 \% \mathrm{MeCN} 0.0-0.5 \mathrm{~min}$, linear gradient of 10-90\% MeCN 0.5-7.0 min, hold 90\% MeCN 7.0-8.0 min, linear gradient of 90-10\% MeCN 8.0-8.1 min, re-equilibrate at 10\% MeCN 8.1-13.25 min)].

Circular dichroism spectroscopy. CD spectra were collected at $25^{\circ} \mathrm{C}$ on a Jasco J-1500 CD spectrometer. Samples were diluted to a calculated concentration of $0.2 \mathrm{mg} / \mathrm{mL}$ in $0.05 \mathrm{M}$ Tris $\mathrm{pH}$ 7.5, 0.15 M NaCl, 0.001 M TCEP. After dilutions, concentrations were determined via absorbance at $\mathrm{A}_{280}$. Buffer subtracted measurements were taken in a $1 \mathrm{~mm}$ cuvette from 260 to $195 \mathrm{~nm}$ and represent an average of five scans. Each sample measurement was normalized for concentration and amino acid count.

Sequence and structural analyses. All sequences were downloaded from either the NCBI database or UniProt, as indicated $(19,46,47)$. Sequence alignments were performed using MAFFT, T-coffee, or BlastP (20, 48, 49). Alignments were visualized using Jalview (50). Homology modeling was performed using the SwissModel web interface $(31,32)$. Structural analyses and figure rendering were done using PyMOL. 
Piper and Struyvenberg et al.

\section{Acknowledgements}

The authors would like to thank the other members of the Amacher and Antos labs for helpful discussions and assistance. JFA was funded by NSF CHE-1904711 and JMA was funded by a Cottrell Scholar Award from the Research Corporation for Science Advancement. KLH was supported by the National Institute of Allergy and Infectious Disease, Award Number F32AI145111. In addition, IMP received an Elwha Undergraduate Summer Research Award and DAJ received a Joseph \& Karen Morse Student Research in Chemistry Fellowship to fund summer research. Start-up funds from Western Washington University also contributed to this project.

\section{Author contributions}

IMP, SAS, JDV, KLH, JMA, and JFA designed the experiments. IMP, SAS, JDV, DAJ, MG, JES, HMK, and KLH performed experiments. KJ and EB contributed solid phase peptide synthesis to the project. IMP, SAS, JDV, DAJ, MG, KLH, JMA, and JFA contributed to data analysis. JMA and JFA wrote the manuscript. IMP, JDV, MG, KLH, JMA, and JFA prepared the figures. All authors contributed to editing of the manuscript.

\section{Declaration of interests}

The authors declare no competing interest. 
Piper and Struyvenberg et al.

Table 1. The amino acid identities of the SrtA residues that are involved in $\beta 7-\beta 8$ loop-mediated interactions. Table 1 includes the amino acid positions in the wild-type proteins of all SrtA proteins discussed.

\begin{tabular}{|l|c|c|c|c|c|c|c|}
\hline & \multicolumn{3}{|c|}{ Interaction (1) } & \multicolumn{2}{c|}{ Interaction (2) } & \multicolumn{3}{c|}{ Interaction (3) } \\
\hline & H-bond 1 & H-bond 2 & $\beta 6^{-2}$ & $\beta 7-\beta 8^{-1}$ & $\beta 4-\beta 5^{+2}$ & $\beta 4-\beta 5^{+3}$ & $\beta 7-\beta 8^{+3}$ \\
\hline B. anthracis & $\mathrm{S} 189^{\mathrm{a}}$ & $\mathrm{D} 191^{\mathrm{a}}$ & $\mathrm{E} 165$ & $\mathrm{~K} 195$ & $\mathrm{M} 128$ & $\mathrm{~S} 129$ & $\mathrm{~V} 190$ \\
\hline S. aureus & $\mathrm{N} 188$ & $\mathrm{~T} 191$ & $\mathrm{D} 160$ & $\mathrm{~K} 196$ & $\mathrm{~F} 122$ & $\mathrm{I} 123$ & $\mathrm{Y} 187$ \\
\hline E. faecalis & $\mathrm{D} 202$ & $\mathrm{~T} 206$ & $\mathrm{~K} 177$ & $\mathrm{~T} 207$ & $\mathrm{~T} 140$ & $\mathrm{E} 141$ & $\mathrm{~L} 203$ \\
\hline L. lactis & $\mathrm{D} 208$ & $\mathrm{~T} 212$ & $\mathrm{~V}^{+} 183^{\mathrm{b}}$ & $\mathrm{H} 213$ & $\mathrm{M} 142$ & $\mathrm{~T} 143$ & $\mathrm{~A} 209$ \\
\hline L. monocytogenes & $\mathrm{D} 189$ & $\mathrm{~T} 194$ & $\mathrm{~T} 166^{\mathrm{c}}$ & $\mathrm{K} 196$ & $\mathrm{M} 129$ & $\mathrm{R} 130$ & $\mathrm{P} 191$ \\
\hline S. oralis & $\mathrm{D} 213$ & $\mathrm{~T} 217$ & $\mathrm{R} 188$ & $\mathrm{E} 218$ & $\mathrm{I} 147$ & $\mathrm{~F} 148$ & $\mathrm{Y} 214$ \\
\hline S. pneumoniae & $\mathrm{D} 209$ & $\mathrm{~T} 213$ & $\mathrm{R} 184$ & $\mathrm{E} 214$ & $\mathrm{I} 143$ & $\mathrm{~F} 144$ & $\mathrm{~L} 210$ \\
\hline S. suis & $\mathrm{D} 208$ & $\mathrm{~T} 212$ & $\mathrm{I} 183^{\mathrm{d}}$ & $\mathrm{Q} 213$ & $\mathrm{I} 142$ & $\mathrm{~F} 143$ & $\mathrm{Y} 209$ \\
\hline
\end{tabular}

${ }^{\mathrm{a}}$ B. anthracis SrtA: Although D191 appears to be positioned to interact with S189, the NMR structure PDB ID 2RUI (ordered loop in the baSrtA bound state) does not show hydrogen bonding between these residues in any of the 20 states.

${ }^{\mathrm{b}}$ L. lactis SrtA: It appears more likely that $\mathrm{H} 213$ interacts with $\mathrm{T} 185$, the $\beta 6-\beta 7^{+1}$ residue, based on a homology model (template: 4TQX, data not shown).

${ }^{\circ} L$. monocytogenes SrtA: In the crystal structure (5HU4), T166 and K196 are not interacting.

${ }^{\mathrm{d} S}$. suis SrtA: It appears more likely that Q213 interacts with $\mathrm{S} 185, \beta 6-\beta 7^{+1}$ residue, based on a homology model (template: 3FN5, data not shown). In the other proteins this position is: B. anthracis (T167), S. aureus (K162), E. faecalis (E179), L. lactis (T185), L. monocytogenes (D168), S. oralis (T190), and S. pneumoniae (T186). 
Piper and Struyvenberg et al.

\section{References}

1. Spirig, T., Weiner, E. M., and Clubb, R. T. (2011) Sortase enzymes in Gram-positive bacteria. Mol. Microbiol. 82, 1044-1059

2. Jacobitz, A. W., Kattke, M. D., Wereszczynski, J., and Clubb, R. T. (2017) Sortase transpeptidases: structural biology and catalytic mechanism. Adv. Protein Chem. Struct. Biol. 109, 223-264

3. Antos, J. M., Truttmann, M. C., and Ploegh, H. L. (2016) Recent advances in sortasecatalyzed ligation methodology. Curr. Opin. Struct. Biol. 38, 111-118

4. Di Girolamo, S., Puorger, C., Castiglione, M., Vogel, M., Gébleux, R., Briendl, M., Hell, T., Beerli, R. R., Grawunder, U., and Lipps, G. (2019) Characterization of the housekeeping sortase from the human pathogen Propionibacterium acnes: first investigation of a class $\mathrm{F}$ sortase. Biochem. J. 476, 665-682

5. Bradshaw, W. J., Davies, A. H., Chambers, C. J., Roberts, A. K., Shone, C. C., and Acharya, K. R. (2015) Molecular features of the sortase enzyme family. FEBS J. 282, 2097-2114

6. Marraffini, L. A., DeDent, A. C., and Schneewind, O. (2006) Sortases and the Art of Anchoring Proteins to the Envelopes of Gram-Positive Bacteria. Microbiol. Mol. Biol. Rev. 70, 192-221

7. Mazmanian, S. K., Liu, G., Ton-That, H., and Schneewind, O. (1999) Staphylococcus aureus sortase, an enzyme that anchors surface proteins to the cell wall. Science 285, 760-763

8. Chen, I., Dorr, B. M., and Liu, D. R. (2011) A general strategy for the evolution of bondforming enzymes using yeast display. Proc. Natl. Acad. Sci. USA 108, 11399-11404

9. Clancy, K. W., Melvin, J. A., and McCafferty, D. G. (2010) Sortase transpeptidases: insights into mechanism, substrate specificity, and inhibition. Biopolymers 94, 385-396

10. Kruger, R. G., Otvos, B., Frankel, B. A., Bentley, M., Dostal, P., and McCafferty, D. G. (2004) Analysis of the substrate specificity of the Staphylococcus aureus sortase transpeptidase SrtA. Biochemistry 43, 1541-1551

11. Dorr, B. M., Ham, H. O., An, C., Chaikof, E. L., and Liu, D. R. (2014) Reprogramming the specificity of sortase enzymes. Proc. Natl. Acad. Sci. USA 111, 13343-13348

12. Bentley, M. L., Lamb, E. C., and McCafferty, D. G. (2008) Mutagenesis studies of substrate recognition and catalysis in the sortase A transpeptidase from Staphylococcus aureus. J. Biol. Chem. 283, 14762-14771

13. Piotukh, K., Geltinger, B., Heinrich, N., Gerth, F., Beyermann, M., Freund, C., and Schwarzer, D. (2011) Directed evolution of sortase A mutants with altered substrate selectivity profiles. J. Am. Chem. Soc. 133, 17536-17539

14. Schmohl, L., Bierlmeier, J., Gerth, F., Freund, C., and Schwarzer, D. (2017) Engineering sortase A by screening a second-generation library using phage display. J Pept Sci 23, 631635

15. Bentley, M. L., Gaweska, H., Kielec, J. M., and McCafferty, D. G. (2007) Engineering the substrate specificity of Staphylococcus aureus Sortase A. The beta6/beta7 loop from SrtB confers NPQTN recognition to SrtA. J. Biol. Chem. 282, 6571-6581

16. Suree, N., Liew, C. K., Villareal, V. A., Thieu, W., Fadeev, E. A., Clemens, J. J., Jung, M. E., and Clubb, R. T. (2009) The structure of the Staphylococcus aureus sortase-substrate complex reveals how the universally conserved LPXTG sorting signal is recognized. J. Biol. Chem. 284, 24465-24477

17. Ton-That, H., Mazmanian, S. K., Alksne, L., and Schneewind, O. (2002) Anchoring of 
Piper and Struyvenberg et al.

surface proteins to the cell wall of Staphylococcus aureus. Cysteine 184 and histidine 120 of sortase form a thiolate-imidazolium ion pair for catalysis. J. Biol. Chem. 277, 7447-7452

18. Wójcik, M., Szala, K., van Merkerk, R., Quax, W. J., and Boersma, Y. L. (2020) Engineering the specificity of Streptococcus pyogenes sortase A by loop grafting. Proteins 88, 13941400

19. UniProt Consortium (2019) UniProt: a worldwide hub of protein knowledge. Nucleic Acids Res. 47, D506-D515

20. Katoh, K., Rozewicki, J., and Yamada, K. D. (2017) MAFFT online service: multiple sequence alignment, interactive sequence choice and visualization. Brief. Bioinformatics, bbx108

21. Campen, A., Williams, R. M., Brown, C. J., Meng, J., Uversky, V. N., and Dunker, A. K. (2008) TOP-IDP-scale: a new amino acid scale measuring propensity for intrinsic disorder. Protein Pept. Lett. 15, 956-963

22. Kyte, J., and Doolittle, R. F. (1982) A simple method for displaying the hydropathic character of a protein. J. Mol. Biol. 157, 105-132

23. Schwartz, G. W., Zhou, Y., Petrovic, J., Fasolino, M., Xu, L., Shaffer, S. M., Pear, W. S., Vahedi, G., and Faryabi, R. B. (2020) TooManyCells identifies and visualizes relationships of single-cell clades. Nat. Methods 17, 405-413

24. Malik, A., and Kim, S. B. (2019) A comprehensive in silico analysis of sortase superfamily. J. Microbiol. 57, 431-443

25. Kattke, M. D., Chan, A. H., Duong, A., Sexton, D. L., Sawaya, M. R., Cascio, D., Elliot, M. A., and Clubb, R. T. (2016) Crystal Structure of the Streptomyces coelicolor Sortase E1 Transpeptidase Provides Insight into the Binding Mode of the Novel Class E Sorting Signal. PLoS One 11, e0167763

26. Zou, Z., Nöth, M., Jakob, F., and Schwaneberg, U. (2020) Designed Streptococcus pyogenes Sortase A Accepts Branched Amines as Nucleophiles in Sortagging. Bioconjug. Chem.

27. Nikghalb, K. D., Horvath, N. M., Prelesnik, J. L., Banks, O. G. B., Filipov, P. A., Row, R. D., Roark, T. J., and Antos, J. M. (2018) Expanding the Scope of Sortase-Mediated Ligations by Using Sortase Homologues. Chembiochem 19, 185-195

28. Schmohl, L., Bierlmeier, J., von Kügelgen, N., Kurz, L., Reis, P., Barthels, F., Mach, P., Schutkowski, M., Freund, C., and Schwarzer, D. (2017) Identification of sortase substrates by specificity profiling. Bioorg. Med. Chem. 25, 5002-5007

29. Kruger, R. G., Dostal, P., and McCafferty, D. G. (2004) Development of a high-performance liquid chromatography assay and revision of kinetic parameters for the Staphylococcus aureus sortase transpeptidase SrtA. Anal. Biochem. 326, 42-48

30. Race, P. R., Bentley, M. L., Melvin, J. A., Crow, A., Hughes, R. K., Smith, W. D., Sessions, R. B., Kehoe, M. A., McCafferty, D. G., and Banfield, M. J. (2009) Crystal structure of Streptococcus pyogenes sortase A: implications for sortase mechanism. J. Biol. Chem. 284, 6924-6933

31. Arnold, K., Bordoli, L., Kopp, J., and Schwede, T. (2006) The SWISS-MODEL workspace: a web-based environment for protein structure homology modelling. Bioinformatics 22, 195-201

32. Bordoli, L., Kiefer, F., Arnold, K., Benkert, P., Battey, J., and Schwede, T. (2009) Protein structure homology modeling using SWISS-MODEL workspace. Nat. Protoc. 4, 1-13

33. Chan, A. H., Yi, S. W., Terwilliger, A. L., Maresso, A. W., Jung, M. E., and Clubb, R. T. (2015) Structure of the Bacillus anthracis Sortase A Enzyme Bound to Its Sorting Signal: A 
bioRxiv preprint doi: https://doi.org/10.1101/2021.03.27.437355; this version posted March 28, 2021. The copyright holder for this preprint (which was not certified by peer review) is the author/funder, who has granted bioRxiv a license to display the preprint in perpetuity. It is made available under aCC-BY-NC-ND 4.0 International license.

Piper and Struyvenberg et al.

FLEXIBLE AMINO-TERMINAL APPENDAGE MODULATES SUBSTRATE ACCESS. J. Biol. Chem. 290, 25461-25474

34. Antos, J. M., Chew, G.-L., Guimaraes, C. P., Yoder, N. C., Grotenbreg, G. M., Popp, M. W.L., and Ploegh, H. L. (2009) Site-specific N- and C-terminal labeling of a single polypeptide using sortases of different specificity. J. Am. Chem. Soc. 131, 10800-10801

35. Raeeszadeh-Sarmazdeh, M., Parthasarathy, R., and Boder, E. T. (2015) Site-specific immobilization of protein layers on gold surfaces via orthogonal sortases. Colloids Surf. B, Biointerfaces 128, 457-463

36. Li, J., Zhang, Y., Soubias, O., Khago, D., Chao, F.-A., Li, Y., Shaw, K., and Byrd, R. A. (2020) Optimization of sortase A ligation for flexible engineering of complex protein systems. J. Biol. Chem. 295, 2664-2675

37. Pritz, S., Wolf, Y., Kraetke, O., Klose, J., Bienert, M., and Beyermann, M. (2007) Synthesis of biologically active peptide nucleic acid-peptide conjugates by sortase-mediated ligation. J. Org. Chem. 72, 3909-3912

38. Guimaraes, C. P., Witte, M. D., Theile, C. S., Bozkurt, G., Kundrat, L., Blom, A. E. M., and Ploegh, H. L. (2013) Site-specific C-terminal and internal loop labeling of proteins using sortase-mediated reactions. Nat. Protoc. 8, 1787-1799

39. Popp, M. W., Artavanis-Tsakonas, K., and Ploegh, H. L. (2009) Substrate filtering by the active site crossover loop in UCHL3 revealed by sortagging and gain-of-function mutations. J. Biol. Chem. 284, 3593-3602

40. Hess, G. T., Guimaraes, C. P., Spooner, E., Ploegh, H. L., and Belcher, A. M. (2013) Orthogonal labeling of M13 minor capsid proteins with DNA to self-assemble end-to-end multiphage structures. ACS Synth. Biol. 2, 490-496

41. Wuethrich, I., Peeters, J. G. C., Blom, A. E. M., Theile, C. S., Li, Z., Spooner, E., Ploegh, H. L., and Guimaraes, C. P. (2014) Site-specific chemoenzymatic labeling of aerolysin enables the identification of new aerolysin receptors. PLoS One 9, e109883

42. Valgardson, J., Cosbey, R., Houser, P., Rupp, M., Van Bronkhorst, R., Lee, M., Jagodzinski, F., and Amacher, J. F. (2019) MotifAnalyzer-PDZ: A computational program to investigate the evolution of PDZ-binding target specificity. Protein Sci. 28, 2127-2143

43. Kang, C.-Y., Huang, I.-H., Chou, C.-C., Wu, T.-Y., Chang, J.-C., Hsiao, Y.-Y., Cheng, C.H., Tsai, W.-J., Hsu, K.-C., and Wang, S. (2020) Functional analysis of Clostridium difficile sortase B reveals key residues for catalytic activity and substrate specificity. J. Biol. Chem. 295, 3734-3745

44. Podracky, C. J., An, C., DeSousa, A., Dorr, B. M., Walsh, D. M., and Liu, D. R. (2021) Laboratory evolution of a sortase enzyme that modifies amyloid- $\beta$ protein. Nat. Chem. Biol. 17, 317-325

45. Pedregosa, F., Varoquaux, G., Gramfort, A., Michel, V., Thirion, B., Grisel, O., Blondel, M., Prettenhofer, P., Weiss, R., Dubourg, V., Vanderplas, J., Passos, A., Cournapeau, D., Brucher, M., Perrot, M., and Duchesnay, É. (2011) Scikit-learn: Machine Learning in Python. J Mach Learn Res 12

46. Magrane, M., and Consortium, U. (2011) UniProt Knowledgebase: a hub of integrated protein data. Database (Oxford) 2011, bar009

47. NCBI Resource Coordinators (2017) Database resources of the national center for biotechnology information. Nucleic Acids Res. 45, D12-D17

48. Notredame, C., Higgins, D. G., and Heringa, J. (2000) T-Coffee: A novel method for fast and accurate multiple sequence alignment. J. Mol. Biol. 302, 205-217 
Piper and Struyvenberg et al.

49. Altschul, S. F., Gish, W., Miller, W., Myers, E. W., and Lipman, D. J. (1990) Basic local alignment search tool. J. Mol. Biol. 215, 403-410

50. Clamp, M., Cuff, J., Searle, S. M., and Barton, G. J. (2004) The Jalview Java alignment editor. Bioinformatics 20, 426-427 
Piper and Struyvenberg et al.

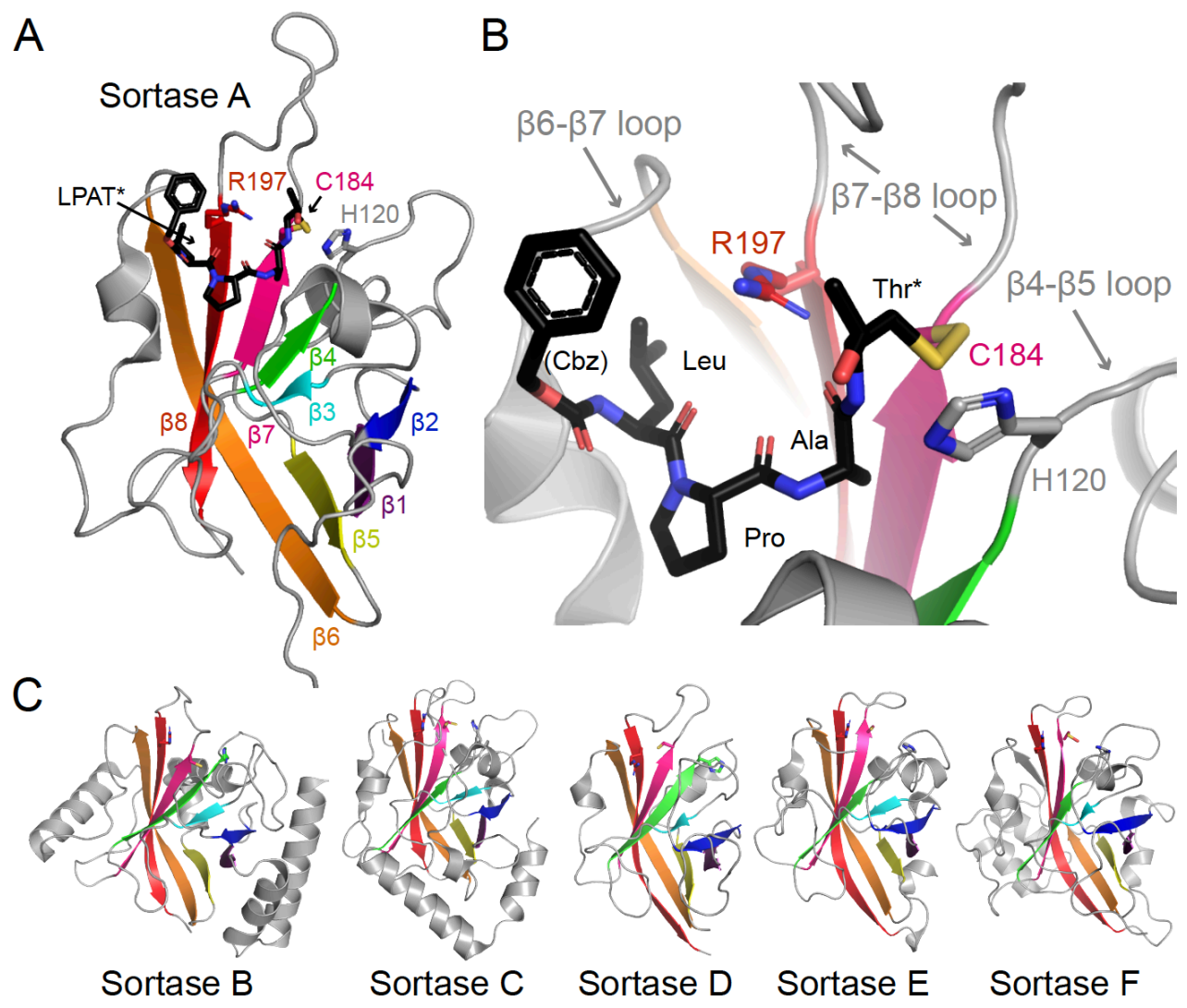

Fig. 1. The sortase-fold is conserved in all classes of bacterial sortases. (A) The peptide-bound structure of $S$. aureus SrtA (saSrtA) is shown in cartoon representation, with $\beta$-strands colored and labeled (PDB ID 2KID) (16). The side chains of the catalytic residues (H120, C184, and R197) are shown as sticks, colored by heteroatom $(\mathrm{O}=\mathrm{red}, \mathrm{N}=$ blue, $\mathrm{S}=$ yellow $)$, and labeled. The peptide analog, Cbz-LPAT*, where Cbz is a carbobenzyloxy protecting group and $\mathrm{T}^{*}$ is $(2 R, 3 S)$-3-amino4-mercapto-2-butanol, is shown as black sticks and colored by heteroatom (16). A zoomed-in version of the active site is shown in (B), with features indicated as in (A). The variable loops are labeled and indicated by gray arrows. (C) The overall sortase-fold is well conserved in proteins of different classes. Here, structures for Class B (PDB ID 1NG5), Class C (3O0P), Class D (2LN7), Class E (5CUW), and Class F (5UUS) sortases are in cartoon, with conserved $\beta$-strands colored as in (A), highlighting the 8-stranded sortase-fold. 
Piper and Struyvenberg et al.
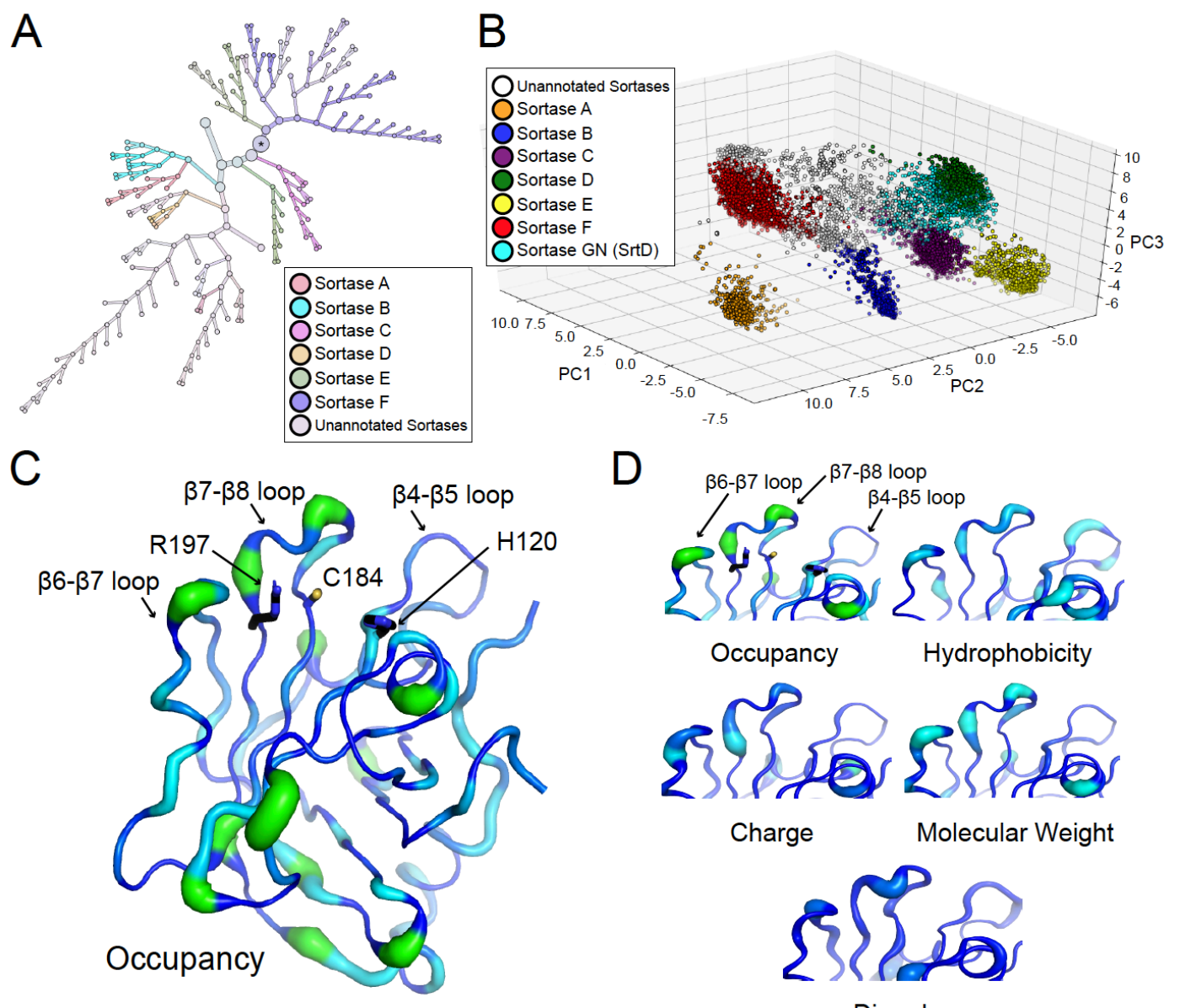

Disorder

Fig. 2. Principal component analysis (PCA) of sortase superfamily reveals sequence variability in structurally-conserved loops. (A) Hierarchical clustering of the sortase superfamily by gaussian mixture model unsupervised classification on the first two components of the singular value decomposition (SVD) matrix distinguishes the known classes of sortases (23). (B) Visualization of a subset of 9,427 of the sortase sequences, scored and filtered with respect to a low number of insertions or deletions (indels) and plotted using principle components 1-3. The sequence is colored by which class of sortase it is annotated as by UniProt, when available. An equivalent plot of all 39,188 sortase sequences is in Fig. S1C. (C-D) The 5 characteristics assigned numerical values in the PCA are visualized by width (from 0 to 1 ) and color (where darker is a value closer to 0 and lighter indicates a value closer to 1 using PyMOL. The Streptococcus pyogenes SrtA structure (PDB ID 3FN5) is used as the model to show variance in a "typical Class A sortase." The catalytic residues (H142, C208, and R216) are shown as side chain sticks and colored by heteroatom. The 3 structurally conserved loops that are discussed in this work are labeled. We focused on variance near the active site here, but notably, there is also a relatively large degree of variance on the other side of the protein (also Fig. S1D). 
Piper and Struyvenberg et al.
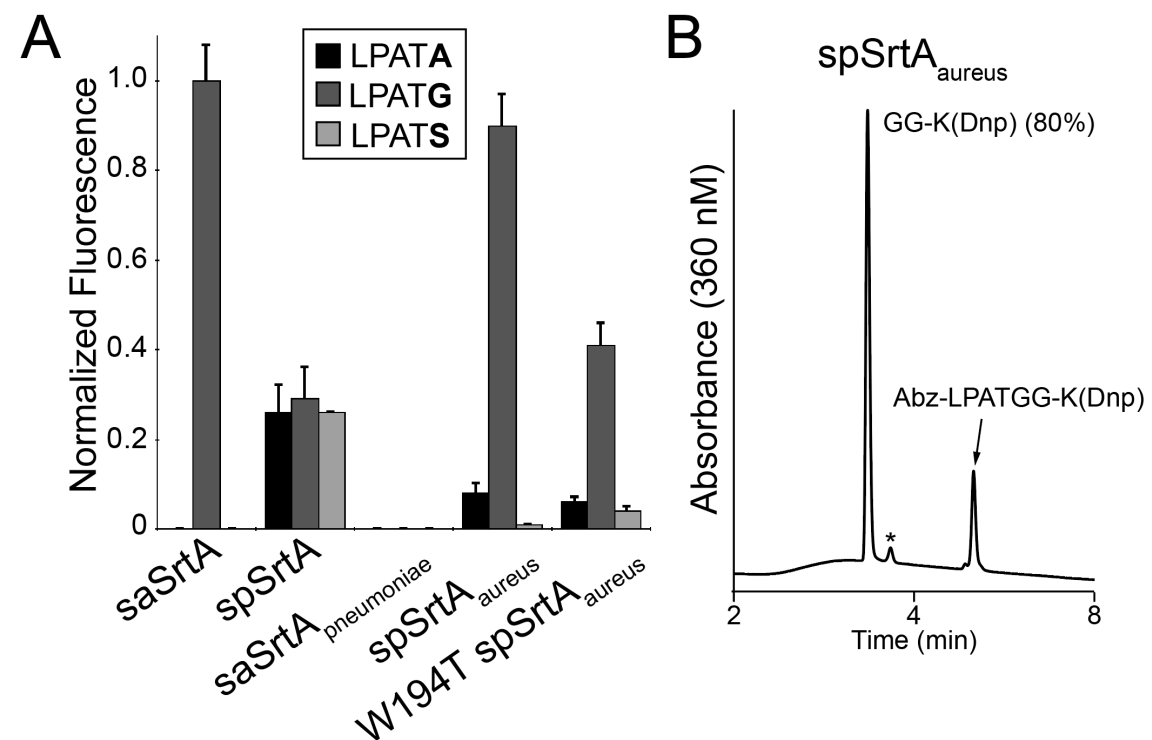

Fig. 3. Interchanging $\beta 7-\beta 8$ loops in class $A$ sortases modulates substrate selectivity and activity for target sequences that vary at position P1' of the canonical LPXTG motif. (A) Comparison of substrate selectivity for wild-type saSrt and spSrtA proteins, as well as $\beta 7-\beta 8$ loop chimeras saSrtA pneumoniae, spSrtA aureus, and W194T spSrtA aureus. Substrate cleavage was monitored via an increase in fluorescence at $420 \mathrm{~nm}$ from reactions of the fluorophore-quencher probes AbzLPATGG-K(Dnp), Abz-LPATAG-K(Dnp), and Abz-LPATSG-K(Dnp) (represented as LPATG, LPATA, and LPATS) in the presence of excess hydroxylamine. Bar graphs represent normalized fluorescence ( \pm standard deviation) from triplicate experiments at the $2 \mathrm{~h}$ reaction timepoint, as compared to saSrtA and the peptide LPATG (raw values are in Table S1). (B) Representative HPLC chromatogram for the reaction of Abz-LPATGG-K(Dnp) and $\mathrm{H}_{2} \mathrm{NOH}$ in the presence of

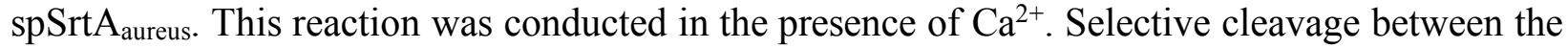
threonine $(\mathrm{T})$ and glycine $(\mathbf{G})$ residues was observed, with an overall conversion of $80 \%$ (* $=$ AbzLPAT-NHOH reaction product. Low peak intensity is due to the weak absorbance of Abz at 360 nm). Also see Fig. S3B-F. 
Piper and Struyvenberg et al.

A
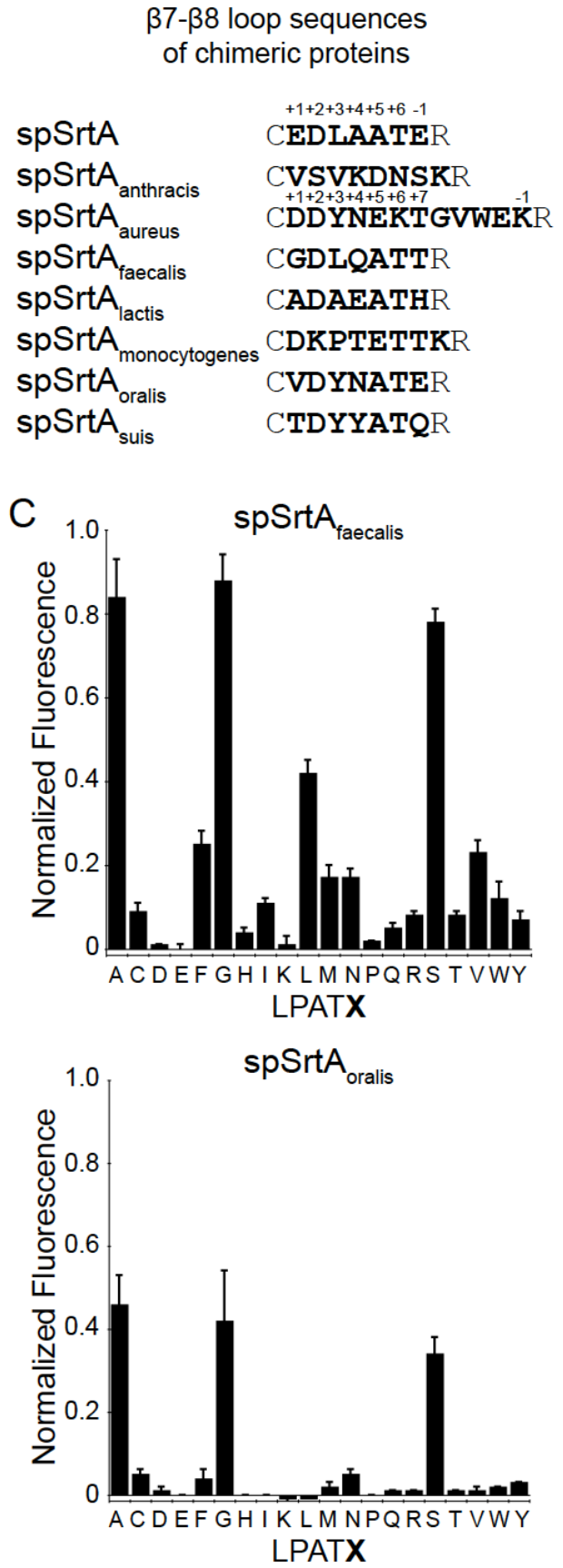

B
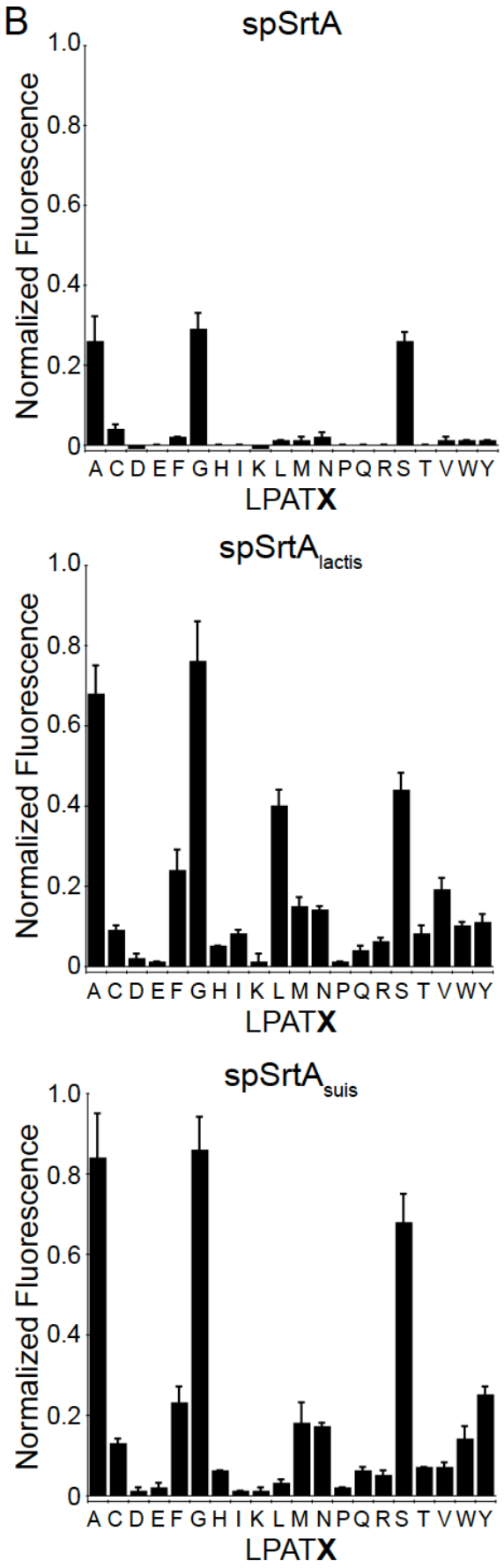

Fig. 4. The sequence of the $\beta$ 7- $\beta 8$ loop dramatically affects selectivity and activity for spSrtA. (A) The $\beta 7-\beta 8$ loop sequences of the chimeric proteins used are listed, with representative numbering for residues in the $\beta 7-\beta 8$ loop labeled for spSrtA and spSrtAaureus. (B-C) Substrate selectivity profiles for wild-type spSrtA (B) and chimeric spSrtA variants (C). Substrate cleavage monitored via an increase in fluorescence at $420 \mathrm{~nm}$ from reactions of fluorophore-quencher probes with the generic structure Abz-LPATXG-K(Dnp) (LPATX) in the presence of hydroxylamine. Bar graphs represent mean normalized fluorescence ( \pm standard deviation) from at least three independent experiments. 
Piper and Struyvenberg et al.

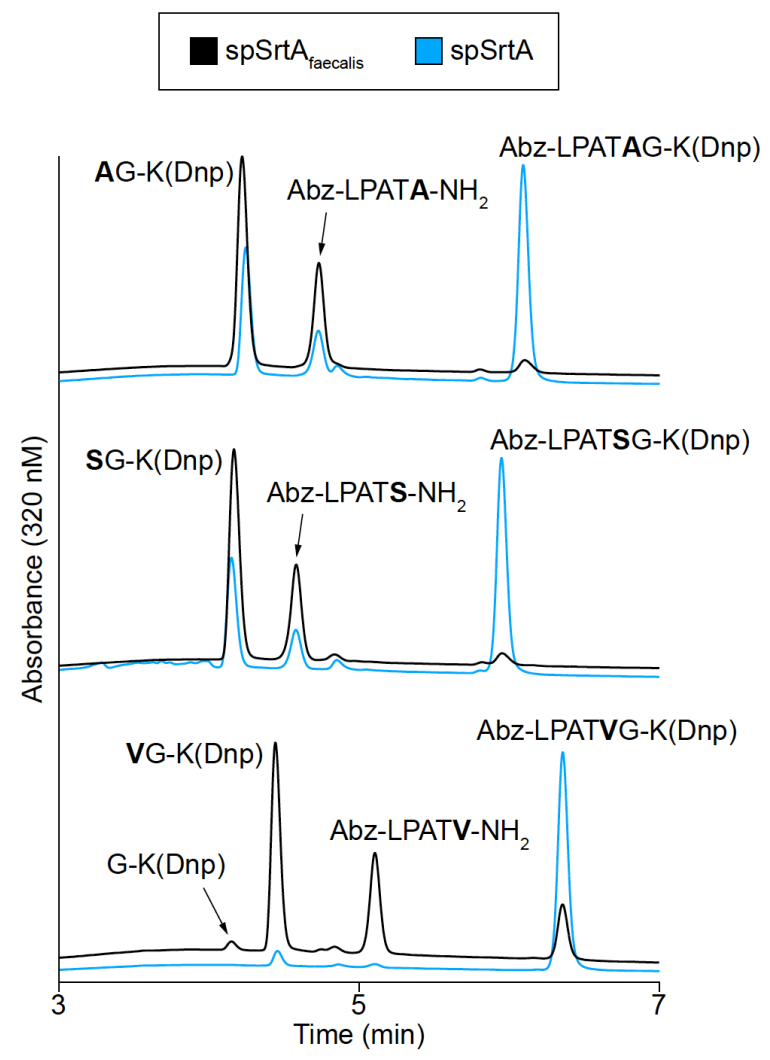

Fig. 5. spSrt $A_{\text {faecalis }}$ outperforms wild-type spSrtA in model amino acid ligation reactions. HPLC chromatograms $(320 \mathrm{~nm})$ for model ligations between Abz-LPATXG-K(Dnp) and excess $\mathrm{X}-\mathrm{NH}_{2}$ nucleophiles catalyzed by spSrtA faecalis (black curves) or wild-type spSrtA (blue curves). Ligations were conducted in the absence of $\mathrm{Ca}^{2+}$. Chromatograms for LPATA/S represent the $3 \mathrm{~h}$ reaction timepoint, and chromatograms for LPATV correspond to the $8 \mathrm{~h}$ timepoint. All peaks identities were confirmed via LC-MS (Table S2). 
Piper and Struyvenberg et al.

A

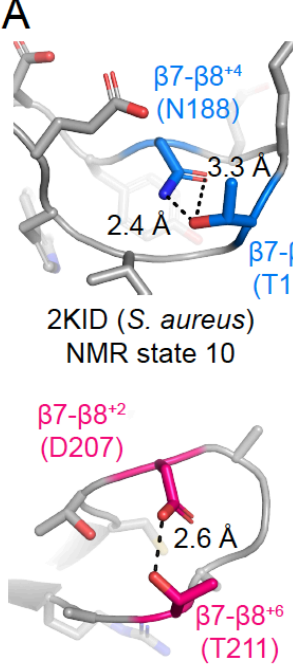

4TQX (S. mutans)

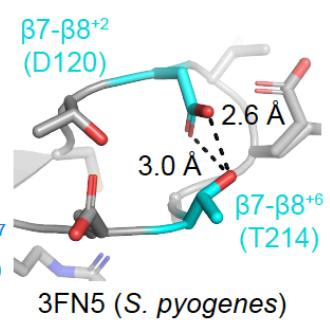

3FN5 (S. pyogenes)

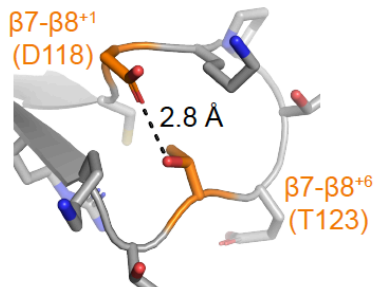

$5 \mathrm{HU} 4$ (L. monocytogenes)
B
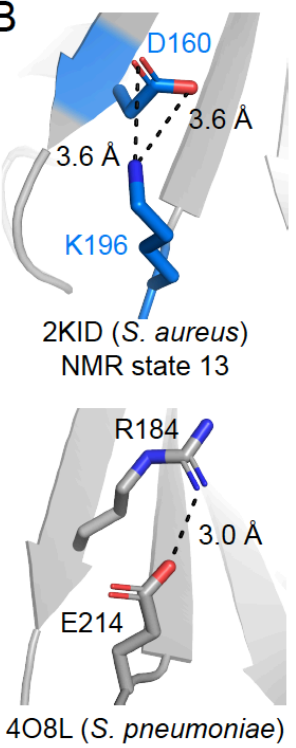

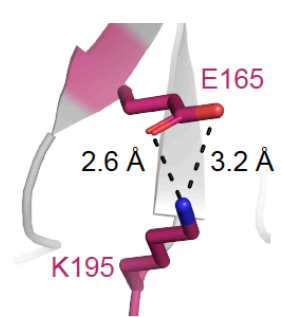

2RUI (B. anthracis) NMR state 1

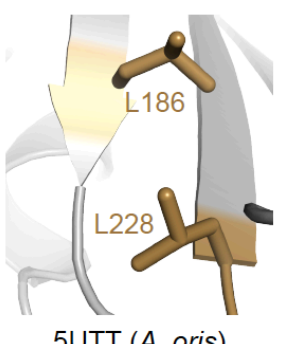

5UTT (A. oris)

C

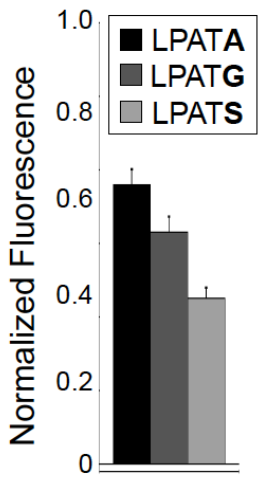

G145E spSrtA $A_{\text {faecalis }}$

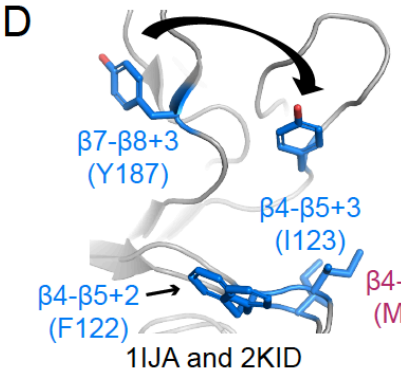

(S. aureus)

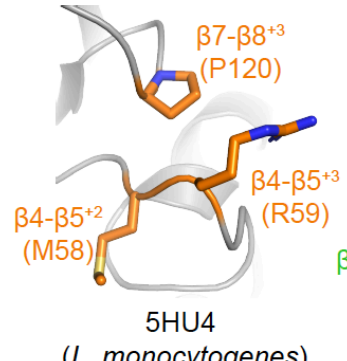

(L. monocytogenes)

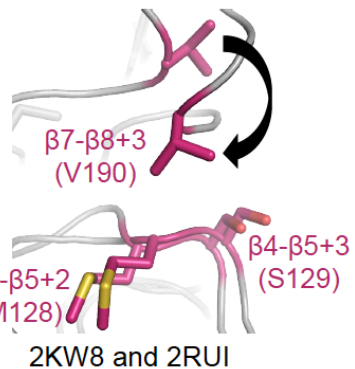

(B. anthracis)

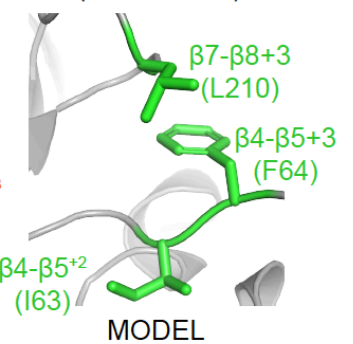

(S. pneumoniae)

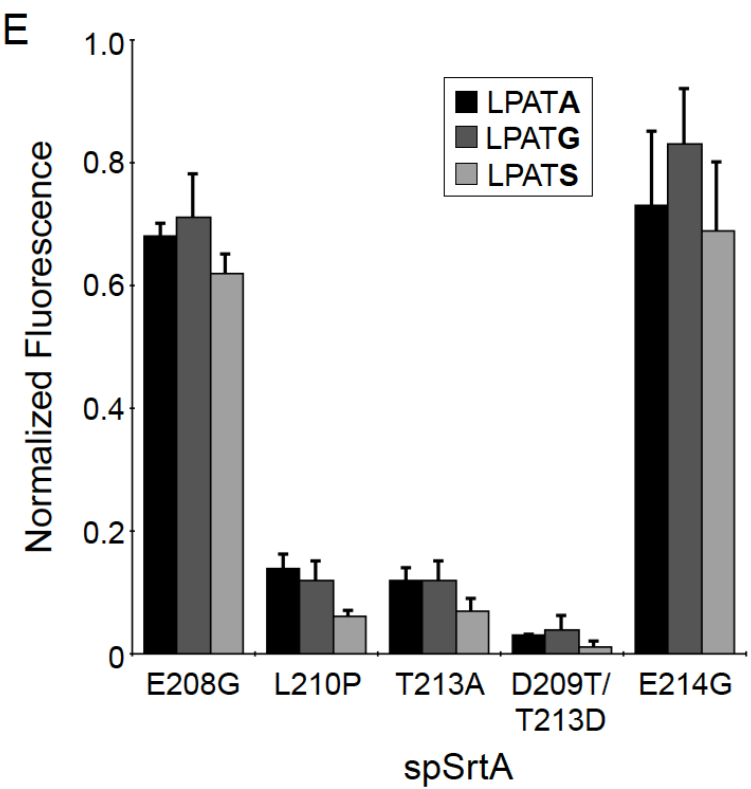

Fig. 6. Residues in the $\beta 7-\beta 8$ loop participate in interactions that affect enzyme activity and selectivity, based on structural analyses and mutagenesis. (A-B, D) All SrtA structures are in gray ribbon. The $\beta 7-\beta 8$ loop side chains are all in stick representation and colored by heteroatom. Residues that participate in hydrogen-bonds have non-gray carbons and are labeled. The hydrogen bonds are shown with a black dashed line, with measurements indicated. For NMR structures (PDB IDs 2KID and 2RUI), the state that was used for the image is labeled. While not all NMR states contained the interaction indicated, in both cases, there were several states that revealed measurements consistent with a non-covalent interaction. Any side chain sticks are colored as described below colored by heteroatom $(\mathrm{O}=\mathrm{red}, \mathrm{N}=\mathrm{blue})$. (A) $\beta 7$ - $\beta 8$ intra-loop hydrogen bond. The structures in this figure are: $S$. aureus SrtA (2KID, blue carbons), S. pyogenes SrtA (3FN5, cyan carbons), $S$. mutans SrtA (4TQX, pink carbons), and L. monocytogenes SrtA (5HU4, orange carbons). (B) Interaction between the $\beta 7-\beta 8$ loop and $\beta 6$ strand. The structures in this figure are: 
Piper and Struyvenberg et al.

S. aureus SrtA (PDB ID 2KID, blue carbons), B. anthracis SrtA (2RUI, dark pink carbons), $S$. pneumoniae SrtA (4O8L, gray carbons), and A. oris SrtA (5UTT, gold carbons). (C) The G145E mutation in $\mathrm{spSrt}_{\text {faecalis }}$ reduces activity for a representative set of target peptides (fluorophorequencher substrate assay conditions identical to those described in Fig. 3). Bar graphs represent normalized fluorescence values ( \pm standard deviation) from three independent experiments. (D) Interaction between the $\beta 7-\beta 8$ and $\beta 4-\beta 5$ loops. The structures in this figure are: $S$. aureus (unbound: 1IJA and bound: 2KID, blue carbons), B. anthracis (unbound: 2KW8 and bound: 2RUI, dark pink carbons), L. monocytogenes (5HU4, orange carbons), and Streptococcus pneumoniae (homology model (Fig. S6), green carbons). The arrows indicate residue movement from the unbound to bound structures. (E) Mutagenesis activity data for spSrtA and G-, S-, and Acontaining peptides. Conditions identical to (C). 
Piper and Struyvenberg et al.
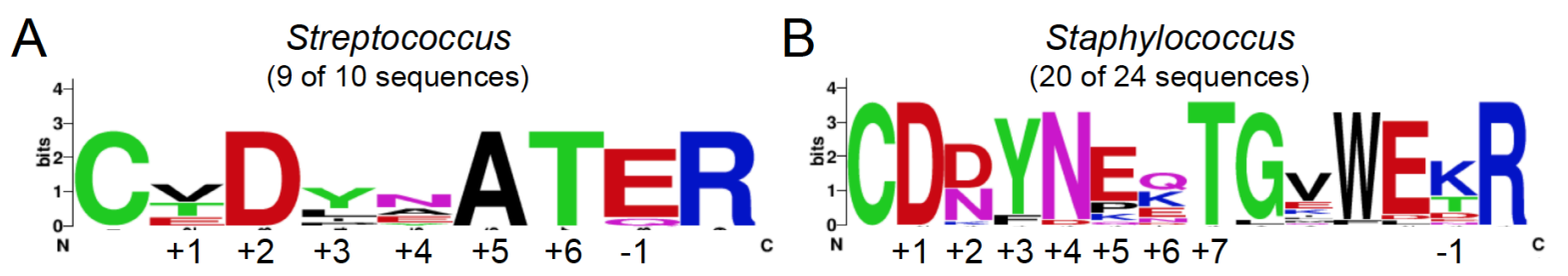

Fig. 7. Conservation in the $\beta$ 7- $\beta 8$ loop sequences of Streptococcus and Staphylococcus Class A sortase sequences. WebLogo analyses of $\beta 7-\beta 8$ loop sequences of the same lengths reveal conservation in the loops for both Streptococcus and Staphylococcus Class A sortases. The catalytic cysteine and arginine residues are also shown to provide reference points for the loop residues, which are labeled as in Fig. 4A. Loop sequences are in Table S3. 
Piper and Struyvenberg et al.

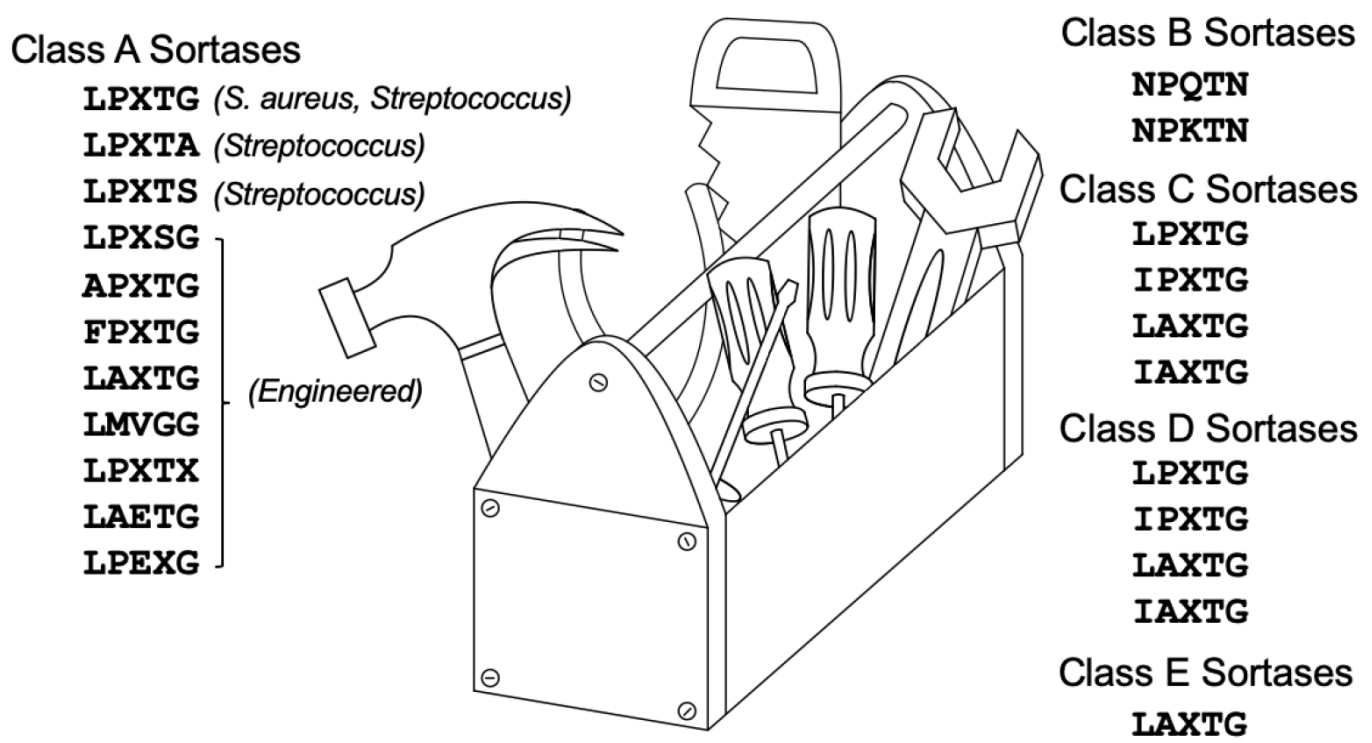

Fig. 8. Building a sortase toolbox for SML experiments. Work from ourselves and others can be used to create a sortase "toolbox" for SML experiments, taking advantage of the various sequence motifs, both endogenous and engineered. Recognition sequences for various sortase subclasses are described in $(5,11,13,14,27,34,44)$. 